\title{
Dynamics of vesicle self-assembly and dissolution
}

\author{
Hiroshi Noguchi ${ }^{a)}$ and Gerhard Gompper ${ }^{\text {b) }}$ \\ Institut für Festkörperforschung, Forschungszentrum Jülich, 52425 Jülich, Germany
}

(Received 9 August 2006; accepted 7 September 2006; published online 27 October 2006)

\begin{abstract}
The dynamics of membranes is studied on the basis of a particle-based meshless surface model, which was introduced earlier [Phys. Rev. E 73, 021903 (2006)]. The model describes fluid membranes with bending energy and - in the case of membranes with boundaries-line tension. The effects of hydrodynamic interactions are investigated by comparing Brownian dynamics with a particle-based mesoscale solvent simulation (multiparticle collision dynamics). Particles self-assemble into vesicles via disk-shaped membrane patches. The time evolution of assembly is found to consist of three steps: particle assembly into discoidal clusters, aggregation of clusters into larger membrane patches, and finally vesicle formation. The time dependence of the cluster distribution and the mean cluster size is evaluated and compared with the predictions of Smoluchowski rate equations. On the other hand, when the line tension is suddenly decreased (or the temperature is increased), vesicles dissolve via pore formation in the membrane. Hydrodynamic interactions are found to speed up the dynamics in both cases. Furthermore, hydrodynamics makes vesicle more spherical in the membrane-closure process. (c) 2006 American Institute of Physics. [DOI: $10.1063 / 1.2358983$ ]
\end{abstract}

\section{INTRODUCTION}

Amphiphilic molecules such as lipids and detergents form a large variety of mesoscopic structures, such as spherical and cylindrical micelles, and bilayer membranes. ${ }^{1-3}$ In particular, bilayer membranes are biologically important as model systems for the plasma membrane and intracellular compartments in living cells. In thermal equilibrium, the morphology of vesicles-closed membranes of usually spherical topology - has been investigated intensively and is now understood very well. ${ }^{3-5}$ Vesicles also exhibit a rich dynamical behavior far from equilibrium. For example, shear flow induces a tank-treading motion of a vesicle ${ }^{6-9}$ and a transition from planar lamellar sheets to multilamellar vesicles. $^{10-12}$ However, in comparison with equilibrium shapes and phase behavior, the phenomena far from thermal equilibrium are much less explored.

Lipids and other surfactant molecules spontaneously assemble and form vesicles in dilute solutions. The selfassembly dynamics was studied experimentally by timeresolved scattering techniques (light, neutron, and $\mathrm{X}$ ray). ${ }^{13-17}$ Typically, the surfactants are found to aggregate into disklike micelles, which grow and transform into a vesicle when their radius exceeds a critical size. The final vesicle size is probably more controlled by kinetics than by thermodynamics. Thus, a detailed understanding of the dynamics of self-assembly is necessary to control the vesicle size.

The solubilization of vesicles is the inverse process of self-assembly. It is well known experimentally that detergents can dissolve lipid membranes into small micelles..$^{13,18-21}$ Nomura et al. ${ }^{21}$ observed several types of

\footnotetext{
${ }^{a)}$ Electronic mail: hi.noguchi@fz-juelich.de

${ }^{b)}$ Electronic mail: g.gompper@fz-juelich.de
}

dynamical behaviors in the lysis of liposomes: opening up by pore formation, inside-out inversion, burst, and vesicle shrinkage with oscillations. Pore-opening and rupture of vesicles were also observed under positive surface tension induced by adhesion to a surface, ${ }^{22}$ optical illumination, ${ }^{22,23}$ or micropipette aspiration. ${ }^{24-26}$

Theoretical models of bilayer membranes can be classified into two groups, depending on whether the bilayer structure is implicitly or explicitly taken into account. In the first group, the bilayer membrane is described as mathematical surface with curvature elasticity. ${ }^{3,4,27}$ This assumption is valid on length scales much larger than the membrane thickness. The information of bilayer properties is only reflected in this case in the values of the elastic parameters. In simulations, a dynamically triangulated mesh ${ }^{27,28}$ is typically employed. The equilibrium properties of membrane with open edges were also studied by this method. ${ }^{29,30}$

In the second group, the bilayer structure is modeled on an atomic ${ }^{31-33}$ or molecular ${ }^{33-41}$ scale, and the solvent is taken into account explicitly ${ }^{31-37}$ or implicitly. ${ }^{38-41}$ The explicit bilayer structure is necessary to study the effects of molecular architecture. However, due to the much larger number of degrees of freedom and thus much larger numerical effort, these models are applicable only for much shorter length and time scales than the curved surface models. In particular, the self-assembly of amphiphilic molecules into micelles, membranes, and vesicles has been simulated only for rather small system sizes. ${ }^{32,35,37,38}$

Recently, we proposed a particle-based meshless membrane model, ${ }^{42}$ which belongs to the first group of membrane models (curved surfaces). The particles possess no internal degrees of freedom - unlike a previous meshless model, ${ }^{43}$ where particles were characterized by an orientation vector. In our model, the curvature potential of a membrane is based 
on the moving least-squares (MLS) method. ${ }^{44,45}$ This model is well suitable to study membrane dynamics accompanied by topological changes.

The aim of this paper is to study the dynamics of selfassembly and dissolution of membranes and vesicles, and to investigate the effect of hydrodynamic interactions. We simulate the self-assembly from a random spatial distribution of monomers into membrane patches, the closure of flat membrane patches into vesicles, and the dissolution of vesicles after rupture. The membrane dynamics without hydrodynamic interactions is studied by Brownian dynamics (BD) -i.e., molecular dynamics with Langevin thermostat. A particle-based mesoscopic solvent model is employed to investigate the membrane dynamics with hydrodynamic interactions. This mesoscale solvent was proposed recently by Malevanets and Kapra ${ }^{46,47}$ and is called multiparticle collision (MPC) dynamics ${ }^{8,48-50}$ or stochastic rotation dynamics (SRD) ${ }^{51-53}$ This method was applied, for example, to polymer dynamics, ${ }^{47,48,50}$ colloid sedimentation, ${ }^{53}$ and vesicles in shear and capillary flows. ${ }^{8,49}$

\section{MODEL AND METHOD}

A membrane consists of $N$ particles, which possess no internal degrees of freedom. The particles interact with each other via a potential

$$
U=\varepsilon\left(U_{\text {rep }}+U_{\text {att }}\right)+k_{\alpha} U_{\alpha},
$$

which consists of a repulsive soft-core potential $U_{\text {rep }}$ with a diameter $\sigma$, an attractive potential $U_{\text {att }}$, and a curvature potential $U_{\alpha}$. All three potentials only depend on the positions $\mathbf{r}_{i}$ of the particles. In this paper, we employ the curvature potential based on the first-order MLS method (model II in Ref. 42). We briefly outline here the simulation technique, since the membrane model and MPC method are explained in detail in Ref. 42 and Refs. 46-48, 51, and 52, respectively.

\section{A. Curvature potential}

The MLS method is a least-squares fit of the membrane shape weighted locally around each particle. ${ }^{42,44,45}$ A Gaussian function with $C^{\infty}$ cutoff $^{42}$ is employed as a weight function

$$
w_{\mathrm{MLS}}\left(r_{i, j}\right)= \begin{cases}\exp \left(\left(r_{i, j} / \sigma\right)^{2} /\left[\left(r_{i, j} / r_{\mathrm{cc}}\right)^{n}-1\right]\right) & \left(r_{i, j}<r_{\mathrm{cc}}\right) \\ 0 & \left(r_{i, j} \geqslant r_{\mathrm{cc}}\right),\end{cases}
$$

where $r_{i, j}$ is the distance between particles $i$ and $j$. This function is smoothly cut off at $r_{i, j}=r_{\mathrm{cc}}$. We use here the parameters $n=12$ and $r_{\mathrm{cc}}=3 \sigma$.

In the first-order MLS method, a plane is fitted to the particle positions by minimizing

$$
\Lambda_{1}\left(\mathbf{r}_{i}\right)=\frac{1}{w_{0}} \sum_{j}\left\{\mathbf{n}\left(\mathbf{r}_{j}-\mathbf{r}_{0}\right)\right\}^{2} w_{\mathrm{MLS}}\left(r_{i, j}\right),
$$

where the sum is over all points (including $i$ itself) and $w_{0}$ $\equiv \Sigma_{j} w_{\mathrm{MLS}}\left(r_{i, j}\right)$ is a normalization factor. The normal vector $\mathbf{n}$ of the plane and the point $\mathbf{r}_{0}$ on the plane are fitting parameters. The minimum of $\Lambda_{1}$ is given by $\Lambda_{1}^{\mathrm{min}}=\lambda_{1}$ when $\mathbf{r}_{0}$ is the weighted center of mass $\mathbf{r}_{G}=\sum_{j} \mathbf{r}_{j} w_{\mathrm{MLS}}\left(r_{i, j}\right) / w_{0}$ and $\mathbf{n}$ is collinear with the eigenvector $\mathbf{u}_{1}$ of the lowest eigenvalue $\lambda_{1}$ of the weighted gyration tensor, $a_{\alpha \beta}=\Sigma_{j}\left(\alpha_{j}-\alpha_{G}\right)\left(\beta_{j}\right.$ $\left.-\beta_{G}\right) w_{\mathrm{MLS}}\left(r_{i, j}\right)$, where $\alpha, \beta=x, y, z$ and $\lambda_{1} \leqslant \lambda_{2} \leqslant \lambda_{3}$.

We define the degree of deviation from a plane, the aplanarity, as

$$
\alpha_{\mathrm{pl}}=\frac{9 D_{w}}{T_{w} M_{w}}=\frac{9 \lambda_{1} \lambda_{2} \lambda_{3}}{\left(\lambda_{1}+\lambda_{2}+\lambda_{3}\right)\left(\lambda_{1} \lambda_{2}+\lambda_{2} \lambda_{3}+\lambda_{3} \lambda_{1}\right)},
$$

where $D_{w}$ and $T_{w}$ are determinant and trace, respectively, and $M_{w}$ is the sum of its three minors, $M_{w}=a_{x x} a_{y y}+a_{y y} a_{z z}$ $+a_{z z} a_{x x}-a_{x y}^{2}-a_{y z}^{2}-a_{z x}^{2}$.

The aplanarity $\alpha_{\mathrm{pl}}$ takes values in the interval $[0,1]$ and represents the degree of deviation from a plane. This quantity acts like $\lambda_{1}$ for $\lambda_{1} \ll \lambda_{2}, \lambda_{3}$, since $\alpha_{\mathrm{pl}} \simeq 9 \lambda_{1} /\left(\lambda_{2}+\lambda_{3}\right)$ in this limit. Therefore, we employ the curvature potential

$$
U_{\alpha}=\sum_{i} \alpha_{\mathrm{pl}}\left(\mathbf{r}_{i}\right),
$$

where $\alpha_{\mathrm{pl}}\left(\mathbf{r}_{i}\right)=0$ when the $i$ th particle has two or less particles within the cutoff distance $r_{i, j}<r_{\mathrm{cc}}$. This potential increases with increasing deviation of the shape of the neighborhood of a particle from a plane, and favors the formation of quasi-two-dimensional membrane aggregates.

\section{B. Attractive and repulsive potentials}

The particles interact with each other in the quasi-twodimensional membrane surface via the potentials $U_{\text {rep }}$ and $U_{\text {att }}$. The particles have an excluded-volume interaction via the repulsive potential

$$
U_{\text {rep }}=\sum_{i<j} \exp \left(-20\left(r_{i, j} / \sigma-1\right)+B\right) f_{\text {cut }}\left(r_{i, j} / \sigma\right),
$$

with $B=0.126$, and a $C^{\infty}$-cutoff function ${ }^{42}$

$$
f_{\text {cut }}(s)= \begin{cases}\exp \left\{A\left(1+1 /\left[\left(|s| s_{\text {cut }}\right)^{n}-1\right]\right)\right\} & \left(s<s_{\text {cut }}\right) \\ 0 & \left(s \geqslant s_{\text {cut }}\right)\end{cases}
$$

is employed. The factor $A$ in Eq. (7) is determined such that $f_{\text {cut }}\left(s_{\text {half }}\right)=0.5$, which implies $A=\ln (2)\left\{\left(s_{\text {cut }} / s_{\text {half }}\right)^{n}-1\right\}$. All orders of derivatives of $f_{\text {cut }}(s)$ are continuous at the cutoff. In Eq. (6), we use the parameters $n=12, A=1$, and $s_{\text {cut }}=1.2$.

A solvent-free membrane or a membrane in an ideal-gastype solvent model such as MPC requires an attractive interaction which mimics the "hydrophobic" interaction. We employ a potential $U_{\text {att }}$ of the local density of particles,

$$
\rho_{i}=\sum_{j \neq i} f_{\text {cut }}\left(r_{i, j} / \sigma\right)
$$

with the parameters $n=12$ and $s_{\text {cut }}=s_{\text {half }}+0.3$ in $f_{\text {cut }}$. Here, $\rho_{i}$ is the number of particles in a sphere whose radius is approximately $r_{\text {att }}=s_{\text {half }} \sigma$. The potential $U_{\text {att }}$ is given by

$$
U_{\text {att }}=\sum_{i} 0.25 \ln \left[1+\exp \left\{-4\left(\rho_{i}-\rho^{*}\right)\right\}\right]-C,
$$

where $C=0.25 \ln \left\{1+\exp \left(4 \rho^{*}\right)\right\}$. For $\rho_{i}<\rho^{*}$, the potential is approximately $U_{\text {att }} \simeq-\rho_{i}$ and therefore acts like a pair poten- 
tial with $U_{\text {att }} \simeq-\sum_{i<j} 2 f_{\text {cut }}\left(r_{i, j} / \sigma\right)$. For $\rho_{i}>\rho^{*}$, this function saturates to the constant $-C$. Thus, it is a pairwise potential with cutoff at densities higher than $\rho_{i}>\rho^{*}$. We used the parameters $r_{\text {att }} / \sigma=1.8$ and $\rho^{*}=6$.

\section{Simulation methods: BD and MPC}

We simulated the membrane in the $N V T$ ensemble (constant particle number $N$, volume $V$, and temperature $T$ ) with periodic boundary conditions in a cubic box with side length $L$. In $\mathrm{BD}$, the motion of particles follows an underdamped Langevin equation,

$$
m_{\mathrm{mb}} \frac{d^{2} \mathbf{r}_{i}}{d t^{2}}=-\zeta \frac{d \mathbf{r}_{i}}{d t}+\mathbf{g}_{i}(t)-\frac{\partial U}{\partial \mathbf{r}_{i}}
$$

where $m_{\mathrm{mb}}$ is the mass of a membrane particle, $\zeta$ the friction coefficient, and $\mathbf{g}_{i}(t)$ a Gaussian white noise which obeys the fluctuation-dissipation theorem. We use $m_{\mathrm{mb}}=1, \zeta=1$, and the temperature $k_{\mathrm{B}} T=1$, where $k_{\mathrm{B}}$ is the Boltzmann constant. The Langevin equations are integrated by the leapfrog algorithm ${ }^{54}$ with a time step of $\Delta t=0.005$.

In MPC, the solvent is described by $N_{s}$ pointlike particles of mass $m_{s}$. The algorithm consists of alternating streaming and collision steps. In the streaming step, the particles move ballistically and the position of each particle $\mathbf{r}_{i}$ is updated according to $\mathbf{r}_{i}\left(t+\Delta t_{\mathrm{CD}}\right)=\mathbf{r}_{i}(t)+\mathbf{v}_{i}(t) \Delta t_{\mathrm{CD}}$, where $\mathbf{v}_{i}$ is the velocity of particle $i$ and $\Delta t_{\mathrm{CD}}$ is the time interval between collisions. In the collision step, the particles are sorted into cubic boxes of lattice constant $\sigma$. The collision step consists of a stochastic rotation of the relative velocities of each particle in a box, $\mathbf{v}_{i}^{(\text {new })}(t)=\mathbf{v}_{\text {c.m. }}(t)+\boldsymbol{\Omega}\left(\mathbf{v}_{i}(t)\right.$ $-\mathbf{v}_{\text {c.m. }}(t)$ ), where $\mathbf{v}_{\text {c.m. }}$ is the velocity of the center of mass of all particles in the box. The matrix $\boldsymbol{\Omega}$ rotates velocities by the angle $\pi / 2$ around an axis, which is chosen randomly for each box. The motions of membrane particles are integrated by the velocity-Verlet algorithm with time step $\Delta t_{\mathrm{MD}}$ $=0.005$, and the membrane particles are included in the MPC collision procedure. ${ }^{47}$

Small mean free paths $\lambda$ are required to obtain large Schmidt numbers, ${ }^{48}$ where $\lambda=\Delta t_{\mathrm{CD}} \sqrt{k_{\mathrm{B}} T / m_{s}}$. Therefore, we choose $\lambda / \sigma=0.1$. We mainly use solvent density $\rho$ $=10 m_{s} / \sigma^{3}$ and the mass of membrane particles $m_{\mathrm{mb}}=\rho \sigma^{3}$ $=10 m_{s}$. The solvent viscosity is $\eta_{0}=5.4 \sqrt{m_{s} k_{\mathrm{B}} T} / \sigma^{2}$ with these parameters. ${ }^{52}$ In order to study the effects of solvent and membrane viscosities on the closure dynamics of vesicles, we also employ the lower solvent viscosity $\eta_{0}$ $=1.9 \sqrt{m_{s} k_{\mathrm{B}} T} / \sigma^{2}$ with $\rho=4 m_{s} / \sigma^{3}$ (but still $m_{\mathrm{mb}}=10 m_{s}$ ) and $\lambda / \sigma=0.1$, and the higher solvent viscosity $\eta_{0}$ $=10.2 \sqrt{m_{s} k_{\mathrm{B}} T} / \sigma^{2}$ with $\rho=10 m_{s} / \sigma^{3}$ and $\lambda / \sigma=0.05$. We use $L=50 \sigma$ in most MPC simulations, but in some cases also $L$ $=100 \sigma$ in order to check for finite-size effects in the closure dynamics. The results are scaled with the diffusion constant $D_{0}$ of an isolated membrane particle. The diffusion constants $D_{0}$ are estimated by the simulations of isolated monomers without potential interactions; this yields $D_{0} \sqrt{m_{s} / k_{\mathrm{B}} T} / \sigma$ $=0.08323 \pm 0.00006$ and $0.03812 \pm 0.00003$ for the low and high viscosities $\eta_{0} \sigma^{2} / \sqrt{m_{s} k_{\mathrm{B}} T}=1.9$ and 5.4, respectively. The averages and error bars of the time developments are calculated from 10 and 20 independent runs for the self-assembly and closure dynamics, respectively. The same number of independent runs is also used in the investigation of the dissolution dynamics.

\section{Membrane properties}

The equilibrium properties (bending rigidity $\kappa$, surface tension $\gamma$, line tension $\Gamma$, and diffusion constant) were investigated in detail in our previous paper. ${ }^{42}$ The free energy of a fluid membrane on sufficiently large length scales is given by ${ }^{55}$

$$
F=\int\left[\gamma+\frac{\kappa}{2}\left(C_{1}+C_{2}\right)^{2}+\bar{\kappa} C_{1} C_{2}\right] d A+\int \Gamma d s,
$$

where $C_{1}$ and $C_{2}$ are the principal curvatures at each point of the membrane, and $\bar{\kappa}$ is the saddle-splay modulus. The first integral in Eq. (11) extends over the membrane surfaces, and the second over the membrane edges. For membranes of fixed topology without edges, the integral over the Gaussian curvature $C_{1} C_{2}$ is an invariant and their properties are independent of $\bar{\kappa}$. However, the behavior of membranes with edges depends on $\bar{\kappa}^{56,57}$

In the meshless membrane model, $\kappa$ and $\Gamma$ can essentially be varied independently. ${ }^{42}$ The bending rigidity $\kappa$ increases linearly with $k_{\alpha}$ and is almost independent of $\varepsilon$, with $\kappa / k_{\mathrm{B}} T=2.2 k_{\alpha} / k_{\mathrm{B}} T-2.5$ at $\varepsilon / k_{\mathrm{B}} T=4$. On the other hand, the line tension $\Gamma$ increases linearly with $\varepsilon$ and is almost independent of $k_{\alpha}$, with $\Gamma \sigma / k_{\mathrm{B}} T=1.16 \varepsilon / k_{\mathrm{B}} T-0.22$ at $k_{\alpha} / k_{\mathrm{B}} T$ $=10$. The saddle-splay modulus is roughly estimated ${ }^{58}$ as $\bar{\kappa}$ $\simeq-\kappa$.

Furthermore, the simulations described in Ref. 42 show that at $k_{\alpha} / k_{\mathrm{B}} T=10$ and $\varepsilon / k_{\mathrm{B}} T=4$, the membrane particles are found to form a stable vesicle with $\kappa / k_{\mathrm{B}} T=20$ and $\Gamma \sigma / k_{\mathrm{B}} T$ $=4.5$. The area $a_{0}=A_{0} / N$ per particle and the area compression modulus $K_{A}$ of a tensionless membrane are found to vary in the range $a_{0} / \sigma^{2}=1.4-1.5$ and $K_{A} \sigma^{2} / k_{\mathrm{B}} T=20-60$ for $\varepsilon / k_{\mathrm{B}} T=4$ and $k_{\alpha} / k_{\mathrm{B}} T \geqslant 5$.

We calculate the membrane viscosity $\eta_{\mathrm{mb}}$ by performing a molecular dynamics simulation of a flat two-dimensional system with $N=1600$ in shear flow. A velocity field $(\dot{\gamma} y, 0)$ is imposed, from which the viscosity is determined via $\eta_{\mathrm{mb}}$ $=\left\langle P_{x y}\right\rangle / \dot{\gamma}$, where $P_{x y}$ is the $x y$ component of the pressure tensor. ${ }^{36}$ The results are shown in Fig. 1. The simulation results indicate a weak dependence of $\eta_{\mathrm{mb}}$ on the surface tension $\gamma$. This dependence is inversely proportional to the diffusion constant obtained in BD. The effects of membrane viscosity are determined by the dimensionless membrane viscosity $\eta_{\mathrm{mb}}^{*}=\eta_{\mathrm{mb}} / \eta_{0} R_{g}$, where $R_{g}$ is the radius of gyration of a vesicle. In our simulations, the dimensionless membrane viscosity $\eta_{\mathrm{mb}}^{*}$ is varied by the solvent viscosity $\eta_{0}$. For a vesicle with $R_{g}=11 \sigma\left(N=1000\right.$ and $\left.k_{\alpha} / k_{\mathrm{B}} T=10\right), \eta_{\mathrm{mb}}^{*}=1$, 0.34 , and 0.18 at $\varepsilon / k_{\mathrm{B}} T=5\left(1.3,0.46\right.$, and 0.25 at $\left.\varepsilon / k_{\mathrm{B}} T=6\right)$ for the solvent viscosities $\eta_{0} \sigma^{2} / \sqrt{m_{\mathrm{S}} k_{\mathrm{B}} T}=1.9,5.4$, and 10.2, respectively. 


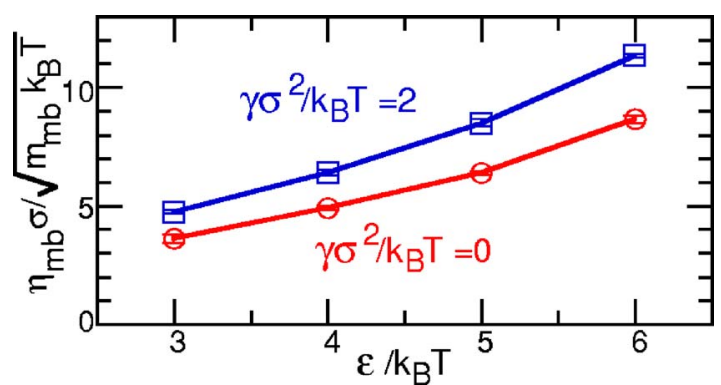

FIG. 1. (Color online) Membrane viscosity $\eta_{\mathrm{mb}}$ as a function of $\varepsilon / k_{\mathrm{B}} T$ for two values of the surface tension, $\gamma \sigma^{2} / k_{\mathrm{B}} T=0$ and $\gamma \sigma^{2} / k_{\mathrm{B}} T=2$, as indicated.

The membrane defined by particles interacting with the potential (1) is partially permeable to the solvent. The permeability $\Lambda$ is defined by $\Delta v=\Lambda \Delta P$, where $\Delta P$ is the pressure difference across membrane, and $\Delta v$ is the difference of the solvent and membrane velocities perpendicular to the membrane. We have estimated the permeability by performing simulations for a flat membrane under a vertical solvent flow, with the center of mass of the membrane fixed in the vertical direction. This implies the results $\Lambda \sqrt{m_{s} k_{\mathrm{B}} T / \sigma^{3}}$ $=0.073$ and 0.042 for $\eta_{0} \sigma^{2} / \sqrt{m_{s} k_{\mathrm{B}} T}=1.9$ and 5.4, respectively.

The relative importance of permeability and hydrodynamics can be assessed by looking at the dynamics of the undulation modes of a nearly planar membrane, whose configurations can be described by a vertical displacement field $h(\mathbf{r}, t)$ from a planar reference state (Monge parametrization). The dynamics is then governed by ${ }^{59}$

$$
\frac{\partial}{\partial t} h(\mathbf{q}, t)=-\left(\Lambda+\frac{1}{4 \eta_{0} q}\right) \kappa q^{4} h(\mathbf{q}, t),
$$

where $h(\mathbf{q}, t)$ is the Fourier transform of $h(\mathbf{r}, t)$. This implies that for wave numbers $q$ smaller than the critical wavelength

$$
q^{*}=\left(4 \eta_{0} \Lambda\right)^{-1}
$$

the dynamics is dominated by hydrodynamics (i.e., length scales larger than $\left.1 / q^{*}\right)$, while for $q>q^{*}$ it is dominated by the permeability. With our parameters given above, we find $\sigma q^{*}$ of order unity, and thus critical wavelengths of a few particle diameters.

\section{FORMATION OF DISKLIKE MICELLES AND VESICLES}

\section{A. Self-assembly}

When the parameters of our model are chosen as described in Sec. II above, particles self-assemble into membranes. An example of this self-assembly process is shown in Fig. 2. We first equilibrate the particles in a gaslike state at low interaction strength $\varepsilon / k_{\mathrm{B}} T=1$, then quench the system to a larger interaction strength $\varepsilon / k_{\mathrm{B}} T=4$ at $t=0$. In BD, we use three densities $\rho_{\mathrm{mb}} \sigma^{3}=0.0038,0.0076$, and 0.015 , corresponding to $N=1000, N=2000$, and $N=4000$ particles in a
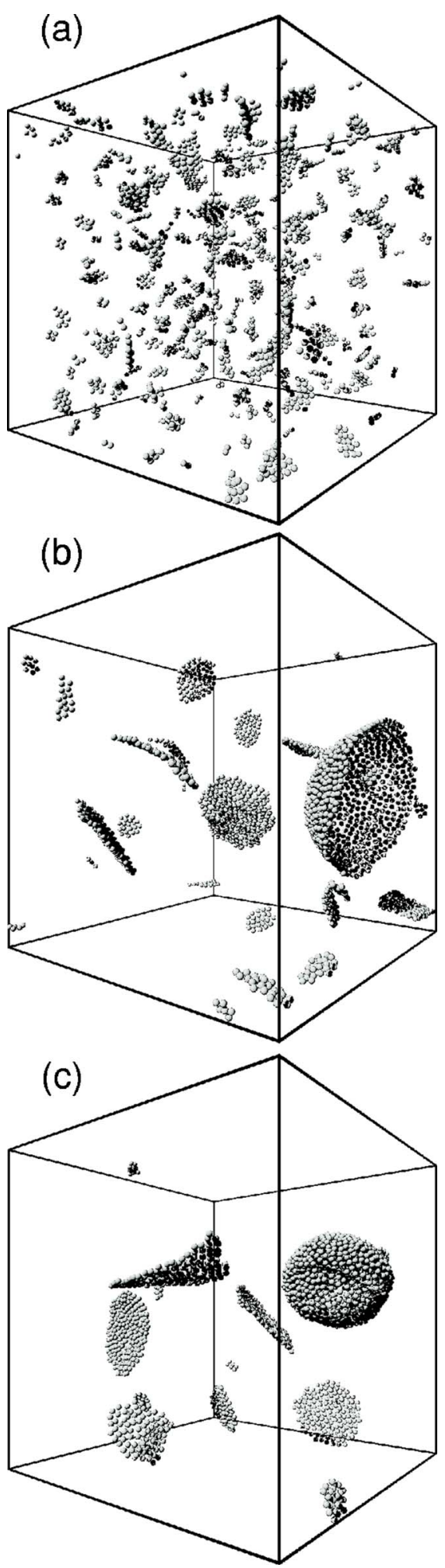

FIG. 2. Snapshots of different stages of the self-assembly process in a BD simulation at the density of membrane particles $\rho_{\mathrm{mb}} \sigma^{3}=0.0076$, with $k_{\alpha} / k_{\mathrm{B}} T=10$ and $\varepsilon / k_{\mathrm{B}} T=4$. (a) $t D_{0} / \sigma^{2}=400$, (b) $t D_{0} / \sigma^{2}=12900$, and (c) $t D_{0} / \sigma^{2}=20000$.

simulation box with $L=64 \sigma$, respectively. We also simulate a smaller box size $L=50 \sigma$ with $\rho_{\mathrm{mb}} \sigma^{3}=0.0076$ for $N=954$ to check for finite-size effects. In MPC, we use the intermediate density $\rho_{\mathrm{mb}} \sigma^{3}=0.0076$ with $L=50 \sigma$ and $N=954$.

Figure 3 shows the time development of the average cluster size $\left\langle n_{c}\right\rangle$, with 


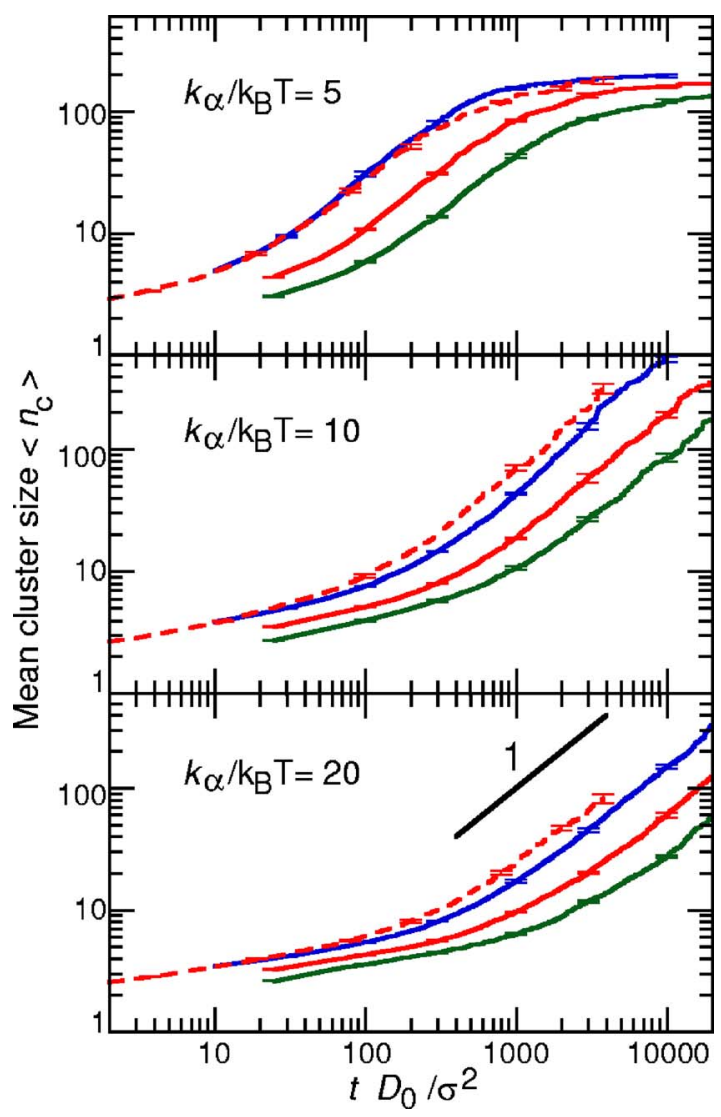

FIG. 3. (Color online) Time development of the mean cluster size $\left\langle n_{c}\right\rangle$ for self-assembly at $\varepsilon / k_{\mathrm{B}} T=4$. The solid lines represent data for $\rho_{\mathrm{mb}} \sigma^{3}$ $=0.0038,0.0076$, and 0.015 (from bottom to top) without hydrodynamic interactions (BD). The dashed lines represent data for $\rho_{\mathrm{mb}} \sigma^{3}=0.0076$ with hydrodynamic interactions (MPC). Error bars are shown at several data points.

$$
n_{c}=\frac{\sum_{i_{c}=1}^{\infty} i_{c}^{2} n_{i}}{\sum_{i_{c}=1}^{\infty} i_{c} n_{i}}
$$

where $i_{c}$ is the size of a cluster and $n_{i}$ is the number of clusters of size $i_{c}$. We define that particles belong to a cluster when their distance is less than $r_{\text {att }}$. The data for BD with $L=50 \sigma$ fall on the same curves as with $L=64 \sigma$ for the same densities (the data are not shown), which implies that finitesize effects are negligible.

The self-assembly consists of three processes: (i) the particle assembly into discoidal clusters, (ii) the discoidal clusters aggregate into larger clusters, and (iii) the large disks close and form vesicles. First, particles assemble into clusters of discoidal shape. In this initial stage, where $\left\langle n_{c}\right\rangle \lesssim 5$, the growth of the average cluster size $\left\langle n_{c}\right\rangle$ is described reasonably well by a power law $t^{0.2}$. Since small clusters with only a few particles have a diameter similar to the membrane thickness, the growth of small clusters will depend on the molecular structures. We therefore expect the growth law $\left\langle n_{c}\right\rangle \sim t^{0.2}$ in this regime to be model dependent.

After most monomers have been incorporated into clusters, the growth mechanism changes to the fusion of clusters. We find in this regime that the average cluster size increases linearly in time, $\left\langle n_{c}\right\rangle \sim t^{1}$. Finally, when the clusters are sufficiently large, they start to form vesicles via a bowl-shaped

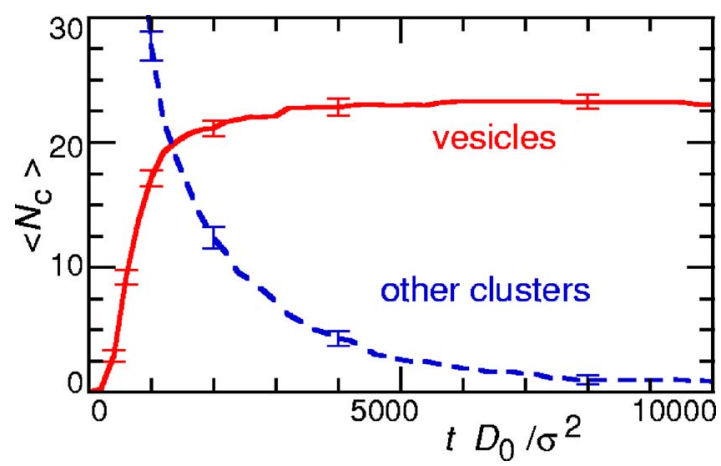

FIG. 4. (Color online) Time development of the average number $\left\langle N_{c}\right\rangle$ of clusters for $\rho_{\mathrm{mb}} \sigma^{3}=0.015, k_{\alpha} / k_{\mathrm{B}} T=5$, and $\varepsilon / k_{\mathrm{B}} T=4$, in a system without hydrodynamic interactions $(\mathrm{BD})$. The solid and dashed lines represent the number of vesicles and the other clusters, respectively. Error bars are shown at several data points.

conformation [see the right cluster in Figs. 2(b) and 2(c)]. Since vesicle fusion is very slow, $\left\langle n_{c}\right\rangle$ saturates and the number of vesicles becomes constant (compare Fig. 4). Thus, the final vesicle size is determined kinetically rather than thermodynamically. This agrees with the experiments of Leng et $a l .{ }^{14,15}$ on the micelle-to-vesicle transition in surfactant solutions.

At larger $k_{\alpha}$, the growth of clusters becomes slower, because the repulsion due to the curvature potential increases for a vertical approach in parallel orientation, as well as for a lateral approach with a finite angle between normal vectors (i.e., for all orientations except for completely parallel disks for a lateral approach). Membrane disks transform into vesicle at cluster sizes $i_{c} \simeq 100$ and 600 for $k_{\alpha}=5$ and 10 , respectively, while no vesicles are observed within the simulation time for $k_{\alpha}=20$.

The assembly dynamics with hydrodynamic interactions (MPC) is faster than for BD (see Fig. 3). The reason must be that hydrodynamic interactions speed up the diffusion of clusters. The diffusion constant of the cluster $i_{c}$ is $D_{i} / D_{0}$ $\sim 1 / R_{\mathrm{dis}}(i) \sim 1 / \sqrt{i_{c}}$ with hydrodynamics, where $R_{\mathrm{dis}}(i) \sim \sqrt{i}$ is the radius of the disk, while $D_{i} / D_{0}=1 / i_{c}$ without hydrodynamic interactions. However, the hydrodynamic interactions do not seem to change the dynamics qualitatively. In both cases, we find $\left\langle n_{c}(t)\right\rangle \sim t$ in the second growth regime.

\section{B. Growth of disklike micelles}

The growth laws of aggregation processes have been studied for decades, and the dynamic power-law scaling $t^{z}$ of spherical aggregation is well understood. ${ }^{60,61}$ However, the growth laws of anisotropic aggregation are still in progress. $^{62,63}$ Our simulation results described in Sec. III A above indicate the dynamic scaling law $n_{c} \sim t^{1}$ for the growth of disklike clusters at long time scales. We want to discuss this growth law on the basis of the Smoluchowski rate equations, ${ }^{64,65}$

$$
\frac{d n_{k}}{d t}=\frac{1}{2} \sum_{j=1}^{k-1} \Omega(j, k-j) n_{j} n_{k-j}-\sum_{j=1}^{\infty} \Omega(j, k) n_{j} n_{k},
$$

where $n_{i}$ is the number density of clusters of size $i$. The first term on the right-hand side of Eq. (15) describes the increase 
of the number of clusters of size $k$ due to binary collisions and fusion of two clusters of smaller sizes $i$ and $j$, while the second term describes the decrease of $k$ clusters due to growth into larger aggregates. In diffusion-limited colloidal aggregation, ${ }^{64,65}$ the kernel $\Omega(i, j)$ has been determined to be

$$
\Omega(i, j)=\left(R_{i}+R_{j}\right)\left(D_{i}+D_{j}\right) \Omega_{0} .
$$

The diffusion constants $D_{i}$ are given by $D_{i}=1 / R_{i}$ and $D_{i}$ $=1 / i$ in systems with and without hydrodynamic interactions, respectively (all prefactors are collected in $\Omega_{0}$ ). With hydrodynamic interactions, $\Omega(i, j)$ is approximately constant. In this case, Eq. (15) has been solved analytically, ${ }^{65}$ and $\left\langle n_{c}\right\rangle$ and all moments $\left\langle\left(\sum i_{c}^{l} n_{i}\right)\right\rangle /\left\langle\left(\sum i_{c}^{l-1} n_{i}\right)\right\rangle$ have been shown to exhibit a $t^{1}$ scaling. This result is in good agreement with our MPC simulations with hydrodynamic interactions. However, with the kernel (16), the Smoluchowski equation predicts a $t^{0.67}$ scaling law for nonhydrodynamics diffusion with $D_{i}$ $=1 / i_{c}$ and $R_{i}=\sqrt{i_{c}}$. This does not agree with the linear time dependence of our BD simulations.

Recently, Leng et al. ${ }^{15}$ proposed another kernel for disklike micelle aggregation,

$$
\Omega(i, j)=R_{\mathrm{dis}}(i)\left\{R_{\mathrm{dis}}(i)+R_{\mathrm{dis}}(j)\right\}\left(D_{i}+D_{j}\right) \Omega_{0} / \xi,
$$

where $\xi$ is a coalescence distance, with $\xi \sim \sigma$ in our model. This is a combination of a reaction-limited kernel ${ }^{66}$ and a factor describing the frequency of coalescence attempts due to diffusion. It is assumed that two disklike micelles are able to merge whenever their edges come closer to each other than the coalescence distance $\xi$. The reaction surface $R_{\text {dis }}(i)$ $\times\left\{R_{\mathrm{dis}}(i)+R_{\mathrm{dis}}(j)\right\}$ is then the area, which the center of the second disk $j$ can cover while the edges are in contact. With the kernel (17), we find the growth laws $\left\langle n_{c}\right\rangle \sim t^{1}$ and $\left\langle n_{c}\right\rangle$ $\sim t^{2}$ without and with hydrodynamic interactions, respectively. The scaling for nonhydrodynamic diffusion agrees with our simulation data for BD. However, in the presence of hydrodynamic interactions, the average cluster size seems to increase much more rapidly than what is observed in the simulations.

There are at least two possible explanations for this discrepancy. The first possibility is that the simulation data are affected by finite-size effects, which can be quite pronounced due to the slow decay of hydrodynamic interactions. The second possibility is that hydrodynamic interactions change the aggregation mechanism. Due to hydrodynamic interactions, the friction coefficient of a disklike membrane and therefore also the diffusion coefficient becomes anisotropic. This implies that membrane disks will approach each other much more easily "horizontally" with both normal vectors pointing in the same direction, so that the reaction area would be $\sigma\left\{R_{\text {dis }}(i)+R_{\text {dis }}(j)\right\}$. This reaction area gives the same kernel of the diffusion-limited aggregation Eq. (16).
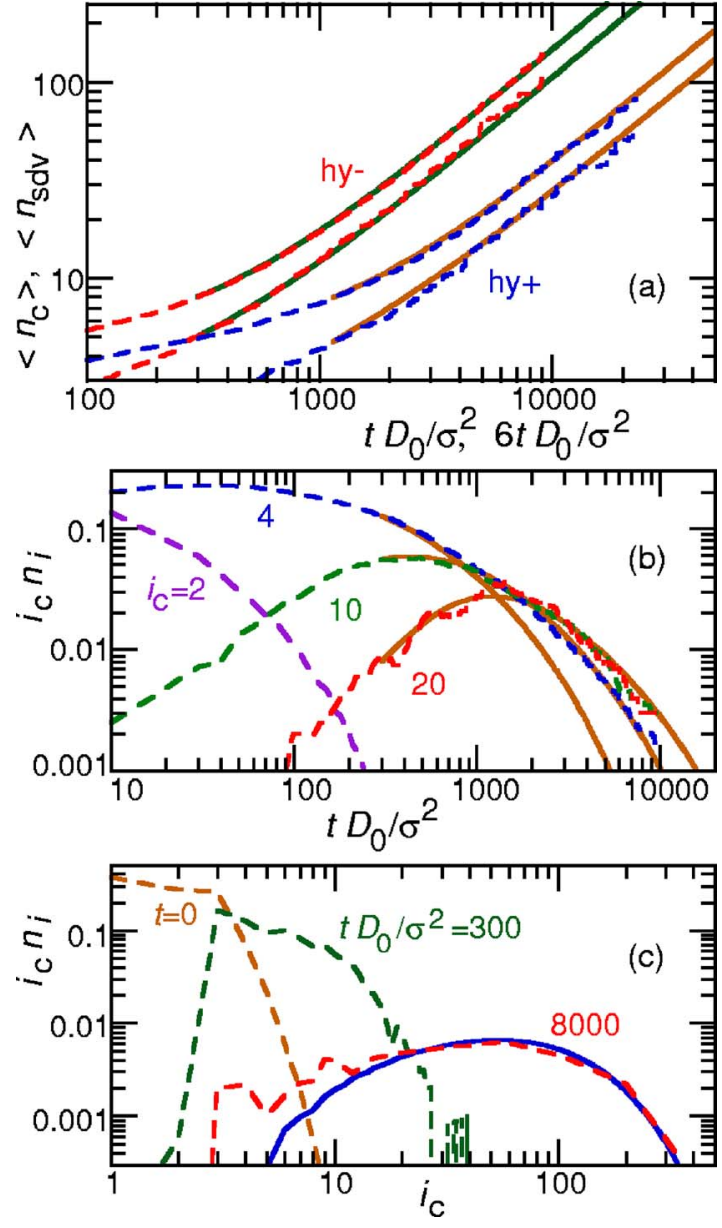

FIG. 5. (Color online) (a) Time development of the average $\left\langle n_{c}\right\rangle$ and the standard deviation $\left\langle n_{\text {sdv }}\right\rangle$ of the cluster-size distribution. The dashed lines represent the simulation data for $k_{\alpha} / k_{\mathrm{B}} T=20$ and $\varepsilon / k_{\mathrm{B}} T=4$ with $\rho_{\mathrm{mb}} \sigma^{3}$ $=0.015(\mathrm{BD})$ or $0.0076(\mathrm{MPC})$. The top two lines represent the BD data for $\left\langle n_{c}\right\rangle$ (upper line) and $\left\langle n_{\text {sdv }}\right\rangle$ (lower line); the bottom two lines represent the MPC data. The solid lines are calculated from Eq. (15) with the kernel (17) for nonhydrodynamic ("hy,$- " \Omega_{0}=0.0014$ ) and the kernel (16) for hydrodynamic diffusion ("hy,$+ " \Omega_{0}=0.005$ ), respectively. The data with hydrodynamics are shifted by a factor of 6 horizontally to avoid an overlap of lines. (b) Time development of the cluster density obtained from BD simulations for the various sizes $i_{c}$, as indicated. (c) Cluster-size distributions obtained from BD simulations at various times $t$, as indicated. In the histogram for $t D_{0} / \sigma^{2}=8000$, data for $i_{c}>10$ are smoothed by averaging over some range of neighboring cluster sizes. The solid lines in (b) and (c) represent the cluster densities and size distribution calculated from Eq. (15) with the simulated distribution at $t D_{0} / \sigma^{2}=300$ as initial condition.

Then, it reproduces our simulation data with hydrodynamic interactions.

A more detailed comparison of the simulation results and the Smoluchowski approach can be made by computing the time-dependent cluster-size distribution functions directly. The results of the average cluster size $\left\langle n_{c}\right\rangle$, the standard deviation $n_{\mathrm{sdv}}^{2} \equiv\left\{\Sigma\left(i_{c}-n_{c}\right)^{2} i_{c} n_{i}\right\} /(N-1)$, and the distributions are displayed in Fig. 5. The initial stage of the aggregation process, where single monomers cluster to form dimers and trimers, is obviously not well described by Smoluchowski kernel for disklike micelles. Therefore, we use the simulated cluster-size distribution at a time, when the number of monomers and dimers has already become negligible, as initial condition in the Smoluchowski equation (15). There is only a 


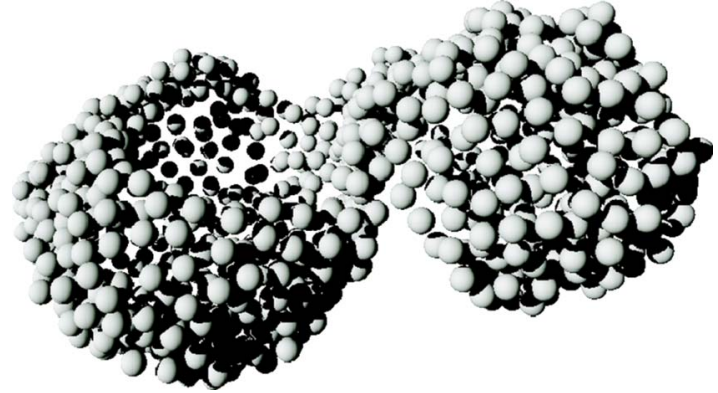

FIG. 6. Snapshot of the S-shaped conformation of a closing membrane for $N=1000, k_{\alpha} / k_{\mathrm{B}} T=2.5$, and $\varepsilon / k_{\mathrm{B}} T=6$.

single fitting parameter, which sets the time scale. The agreement of the results of both approaches is excellent. However, further studies are needed to clarify the aggregation mechanism.

\section{Vesicle closure}

The curvature energies (11) of a spherical vesicle and a flat discoidal membrane are $F_{\mathrm{sph}}=8 \pi(\kappa+\bar{\kappa} / 2)$ and $F_{\text {dis }}$ $=2 \pi R_{\mathrm{dis}} \Gamma$, respectively. Thus, with $R_{\mathrm{dis}}=\sqrt{a_{0} N / \pi}$ and $\bar{\kappa}=$ $-\kappa$ (see Sec. II D), a vesicle has lower free energy than a disk for aggregation numbers $N>N_{0}=\pi(\kappa / \Gamma)^{2} / a_{0}$. Fromherz ${ }^{67}$ calculated the free energy of vesicle closure under the assumption that the membrane shape is a spherical cap. He found that there is a free-energy barrier between spherical and disk shape for $N<4 N_{0}$. Thus, the flat membrane is metastable for $N_{0}<N<4 N_{0}$, but unstable for $N \geqslant 4 N_{0}$. This implies that the critical vesicles sizes for $k_{\alpha}=5,10$, and 20, are $N_{0}=10,43$, and $190\left(4 N_{0}=40,170\right.$, and 760), respectively. These values agree very well with the simulation results.

To investigate the closure dynamics into a vesicle in more detail, we employ a flat square-shaped membrane with a fixed size as initial condition, which quickly relaxes to a nearly planar circular shape. In all simulations, this size is larger than the critical size $4 N_{0}$ predicted by Fromherz. ${ }^{67}$

When the membrane size is much larger than the minimum size for closure, the membrane shapes at large line tension $\Gamma$ resemble the buckled conformations of nearly planar membranes with periodic boundary conditions and decreasing projected area, as they are often observed to be buckled to both sides (with concave and convex regions) with a sine-like deformation [compare Fig. 2(c) of Ref. 42].

For vesicle sizes far above the metastability limit, i.e., $N \gg 4 N_{0}$, the "symmetrically" buckled states often persist and close in both directions via an S-shaped conformation, as shown in Fig. 6. Then, the membrane transforms into two separate vesicles, or into a single vesicle with a piece of a flat membrane inside, which ruptures later in time. The probability of the later pathway depends on the stability of a branching line in the membrane, which decreases with increasing $k_{\alpha}$. Thus, two separate vesicles are formed at $k_{\alpha} / k_{\mathrm{B}} T=5$ and $N=4000$, and a single vesicle is formed at $k_{\alpha} / k_{\mathrm{B}} T=2.5$ and $N=1000$. On the other hand, closer to the metastability limit, "symmetrically" buckled conformations are not observed, and the bowl shape is the only pathway of closure, for example, at $k_{\alpha} / k_{\mathrm{B}} T=5$ and $N=1000$. In this paper, we focus on

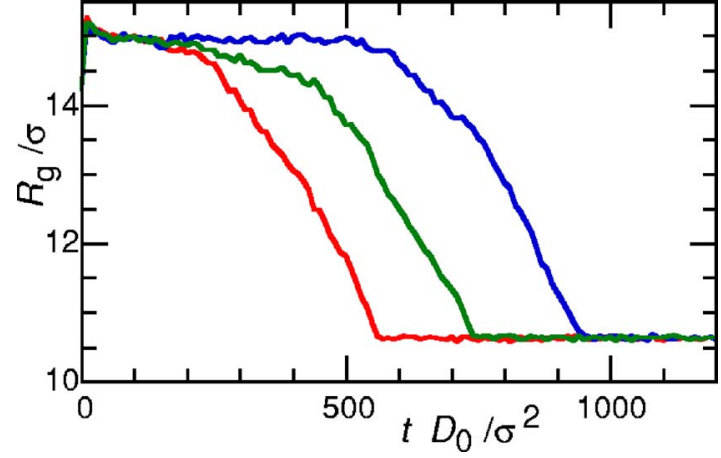

FIG. 7. (Color online) Time development of the radius of gyration $R_{g}$ for the closure of a vesicle from a flat membrane at $N=1000, k_{\alpha} / k_{\mathrm{B}} T=10$, and $\varepsilon / k_{\mathrm{B}} T=5$, as obtained from BD simulations. The lines represent three examples with different realizations of the thermal white noise.

the closing dynamics via a bowl shape $\left(k_{\alpha} / k_{\mathrm{B}} T \geqslant 5\right.$ and $N$ $=1000$ ), since this type of closure occurs in the self-assembly dynamics (compare Sec. III A), because membrane disks close into vesicles as soon as they reach the critical size.

Figure 7 shows three events of vesicle closure (with identical parameters). The initiation of closure is stochastic, determined by a sufficiently large thermal undulation of the nearly planar disk, which subsequently transforms into a bowl shape. The curves of the time-dependent radius of gyration $R_{g}(t)$ are almost parallel for $11 \sigma \leqslant R_{g} \leqslant 13 \sigma$ in Fig. 7. We therefore set the reference time $t_{0}$ at $R_{g}=12 \sigma$, and then average the time developments of $R_{g}$ and the asphericity $\alpha_{\mathrm{sp}}$. Here, the asphericity is the degree of deviation from a spherical shape, ${ }^{68}$

$$
\alpha_{\mathrm{sp}}=\frac{\left(\lambda_{1}-\lambda_{2}\right)^{2}+\left(\lambda_{2}-\lambda_{3}\right)^{2}+\left(\lambda_{3}-\lambda_{1}\right)^{2}}{2 R_{g}^{4}},
$$

where $\lambda_{1} \leqslant \lambda_{2} \leqslant \lambda_{3}$ are the eigenvalues of the gyration tensor and $R_{g}^{2}=\lambda_{1}+\lambda_{2}+\lambda_{3}$. This is a convenient measure to distinguish spherical and discoidal shapes, where $\alpha_{\mathrm{sp}}=0$ for spheres, $\alpha_{\mathrm{sp}}=1$ for thin rods, and $\alpha_{\mathrm{sp}}=0.25$ for thin disks. During closure, $R_{g}$ and $\alpha_{\mathrm{sp}}$ decrease as shown in Fig. 8. The energy difference $U_{\text {att }}-U_{\text {att }}^{\mathrm{eq}}$, where $U_{\text {att }}^{\mathrm{eq}}$ is the value of the vesicle at equilibrium, is mainly caused by the line tension of the membrane edge, and is therefore approximately proportional to the edge length.

In order to investigate the membrane shapes during closure, we show $R_{g} / R_{\text {eq }}$ versus $\alpha_{\text {sp }}$ in Fig. 9, where $R_{\text {eq }}$ is the radius of the closed vesicle at equilibrium. In the simulations, we use the radius of gyration to calculate $R_{\text {eq }}$. The obvious reference state is a spherical cap, the section of sphere, with a decreasing pore opening angle $\theta_{\mathrm{po}}$. In cylindrical coordinates, the spherical cap is represented by $r$ $=R \sin (\theta)$ and $z=R \cos (\theta)$ with $\theta=\left[\theta_{\mathrm{po}}, \pi\right]$. Then, $R_{g}$ and $\alpha_{\mathrm{sp}}$ are given by

$$
\begin{aligned}
& \frac{R_{g}}{R_{\mathrm{eq}}}=\sqrt{\frac{3-\cos \left(\theta_{\mathrm{po}}\right)}{2},} \\
& \alpha_{\mathrm{sp}}=\frac{4 \sin ^{4}\left(\theta_{\mathrm{po}} / 2\right)}{\left(\cos \left(\theta_{\mathrm{po}}\right)-3\right)^{2}},
\end{aligned}
$$

which implies that 


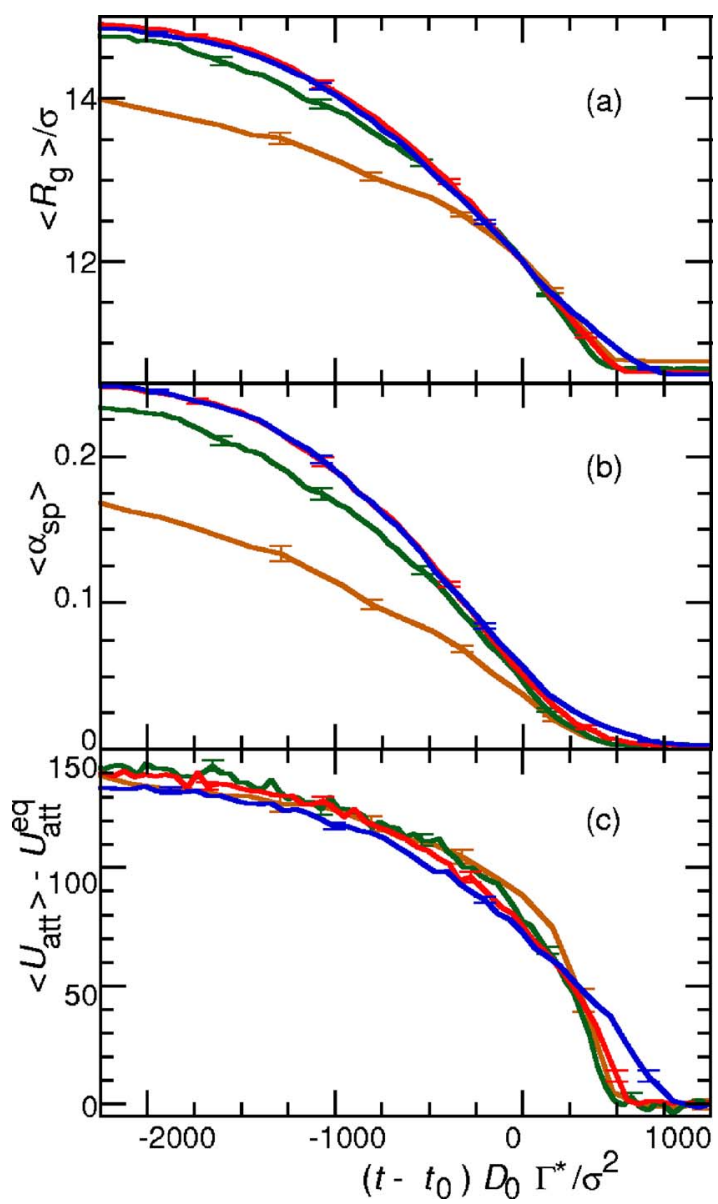

FIG. 8. (Color online) Time development of (a) the radius of gyration $\left\langle R_{g}\right\rangle$, (b) the asphericity $\left\langle\alpha_{\mathrm{sp}}\right\rangle$, and (c) the attractive energy $\left\langle U_{\text {att }}\right\rangle$ for vesicle closure with $N=1000$ and $k_{\alpha} / k_{\mathrm{B}} T=10$. The lines represent data of $\mathrm{BD}$ for $\varepsilon / k_{\mathrm{B}} T=3,4,5$, and 6 (from bottom to top; brown, green, red, and blue). The reference time $t_{0}$ is set at $R_{g} / \sigma=12$; time is rescaled by the dimensionless line tension $\Gamma^{*}=\Gamma \sigma / k_{\mathrm{B}} T$ to reduce the difference in time scales. Error bars are shown at several data points.

$$
\alpha_{\mathrm{sp}}=\left\{1-\left(R_{\mathrm{eq}} / R_{g}\right)^{2}\right\}^{2} .
$$

For small $\Gamma$ or large $\kappa$, the simulated shapes follow the prediction (21) [compare with the bottom line in Fig. 9(c)] very well. With increasing line tension and decreasing bending rigidity, deviation from the spherical-cap shape becomes important, because the deformation force increases and simultaneously the bending resistance decreases. The membrane closes via an oblate ellipsoidal shape at large $\Gamma / \kappa$, since this is a shorter pathway to a closed vesicle state, although it is accompanied by a larger membrane curvature. In addition, since thermal fluctuations of the membrane increase $\left\langle\alpha_{\mathrm{sp}}\right\rangle$, the curves for smaller bending rigidity, $k_{\alpha} / k_{\mathrm{B}} T=5$, are shifted upwards. Note that at small $k_{\alpha}$, the deviation from the spherical-cap shape can become so large that the second derivative of $\alpha_{\mathrm{sp}}\left(\left\langle R_{g}\right\rangle\right)$ changes sign. Therefore, the assumption of the spherical cap is justified for $k_{\alpha} / k_{\mathrm{B}} T>5$ and large $\varepsilon$ within BD.

Hydrodynamic interactions affect not only the time scale but also the membrane shapes during closure. Figures 9(b) and 9(c) show that the membrane is more spherical with the hydrodynamic interactions. The membrane pushes the solvent during the closure; this generates a dynamic pressure in

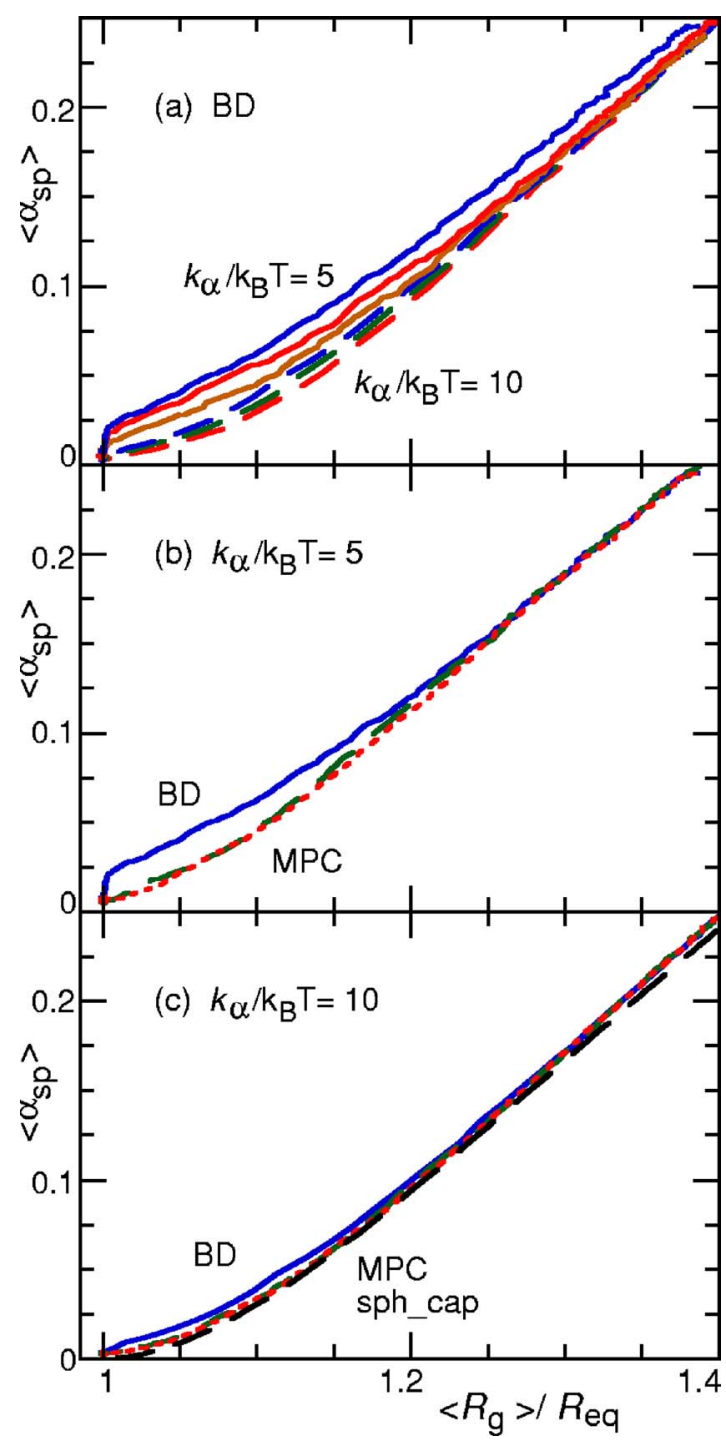

FIG. 9. (Color online) Relation of the average asphericity $\left\langle\alpha_{\mathrm{sp}}\right\rangle$ to the normalized radius of gyration $\left\langle R_{g}\right\rangle / R_{\text {eq }}$ during vesicle closure, for membranes of size $N=1000$. (a) Dependence on $\varepsilon$ without hydrodynamic interactions (BD). The solid lines represent data for $k_{\alpha} / k_{\mathrm{B}} T=5$ with $\varepsilon / k_{\mathrm{B}} T=2.5,3$, and 6 (from bottom to top; brown, red, and blue). The dashed lines represent data for $k_{\alpha} / k_{\mathrm{B}} T=10$ with $\varepsilon / k_{\mathrm{B}} T=3,4$, and 6 (from bottom to top; red, green, and blue). [(b) and (c)] Effect of hydrodynamic interactions for $\varepsilon / k_{\mathrm{B}} T=6$. The solid lines (blue) represent the BD results. The dashed (green) and dotted (red) lines represent the MPC data with $\eta_{\mathrm{mb}}{ }^{*}=0.46$ and 1.3 , respectively. In (c), the spherical-cap result, Eq. (21), is given by the dashed line (black) marked "sph_cap."

the solvent, which makes the membrane more spherical. For the two investigated membrane viscosities, with $\eta_{\mathrm{mb}}^{*}$ of order unity, the effect of the membrane viscosity on the shape is very small, almost negligible. Hydrodynamic interactions also modify the time dependence of $R_{g}$, as shown in Fig. 10. The membrane dynamics depends on both the solvent and membrane viscosities. The characteristic time scale $\tau$ in the regime dominated by the membrane viscosity has been predicted $^{22}$ to increase linearly with membrane viscosity $\eta_{\mathrm{mb}}$ and to decrease as $1 / \Gamma$ with increasing line tension. We therefore postulate that in the more general case, where both solvent and membrane viscosities are important, the time scale can be written as $\tau=\left(\eta_{0} R_{\text {eq }}^{2}+\chi \eta_{\mathrm{mb}} R_{\mathrm{eq}}\right) / \Gamma=\eta_{0}$ $\left(1+\chi \eta_{\mathrm{mb}}^{*}\right) R_{\mathrm{eq}}^{2} / \Gamma$. The $R_{g}(t)$ curves for different viscosities 

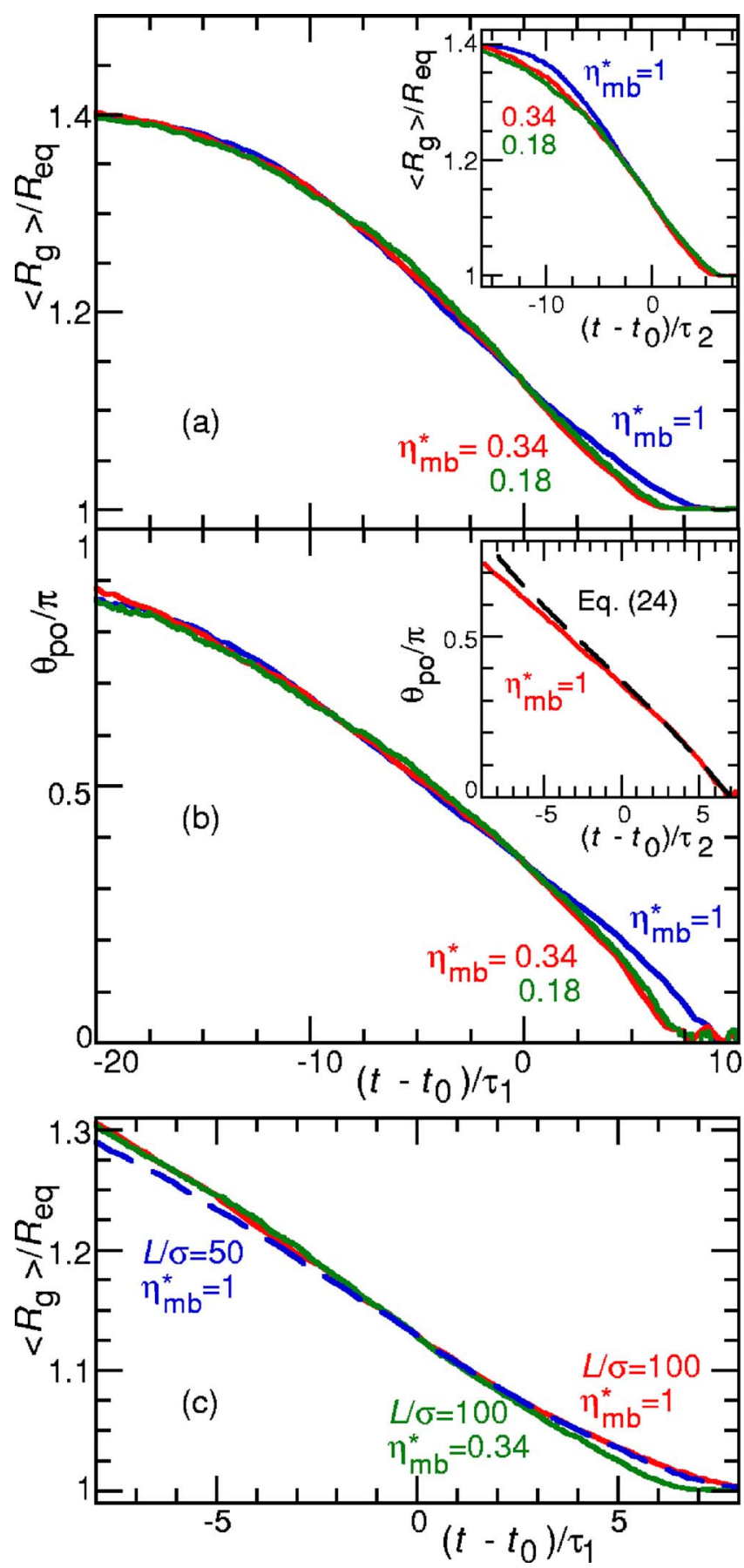

FIG. 10. (Color online) Time development of $[(\mathrm{a})$ and (c) $]$ the radius of gyration $\left\langle R_{g}\right\rangle$ and (b) the pore angle $\theta_{\mathrm{po}}$ for vesicle closure with $N=1000$, $k_{\alpha} / k_{\mathrm{B}} T=10$, and $\varepsilon / k_{\mathrm{B}} T=5$. The angle $\theta_{\mathrm{po}}$ is calculated from $R_{g}$ with Eq. (19). The box size in (a) and (b) is $L / \sigma=50$. The lines represent MPC data with $\eta_{\mathrm{mb}}^{*}=0.18,0.34$, and 1 , as indicated. The time axis is scaled with the characteristic time $\tau_{1}=\eta_{0}\left(1+0.5 \eta_{\mathrm{mb}}^{*}\right) R_{\mathrm{eq}}^{2} / \Gamma$. The insets in (a) and (b) show the same data, but the time axis now scaled with $\tau_{2}=\eta_{0}\left(1+\eta_{\mathrm{mb}}^{*}\right) R_{\mathrm{eq}}{ }^{2} / \Gamma$. The dashed line in the inset of (b) shows the result of Eq. (24) with $\tau=2.6 \tau_{2}$. The solid and dashed lines in (c) represent data for $L / \sigma=100$ and $L / \sigma=50$, respectively.

can be made to coincide in the early and late stages for $\chi$ $\simeq 0.5$ and $\chi \simeq 1$, respectively, as demonstrated in Fig. 10(a). Indeed, the first formation of a bowl-shaped membrane conformation can be expected to be mainly controlled by the solvent viscosity, since the displacements of the membrane are initially perpendicular to the planar reference state, so that little flow of membrane particles is required. However, in the later stage of closure $\left[\theta_{\mathrm{po}}<0.3 \pi\right.$, see Fig. 10(b)], the dynamics should slow down with increasing membrane viscosity, since flow inside the membrane is now unavoidable. Therefore, the importance of membrane viscosity increases as the vesicle closure progresses.

For the box size $L / \sigma=50$, the diameter of the disklike membrane $\left(2 R_{\mathrm{dis}} / \sigma=42\right)$ is not much smaller than the box size. In order to check for finite-size effects, we have therefore simulated vesicle closure for an initially bowl-shaped membrane also for a larger box with $L / \sigma=100$ (and $N$ $=1000, k_{\alpha} / k_{\mathrm{B}} T=10$, and $\left.\varepsilon / k_{\mathrm{B}} T=5\right)$. Figure 10(c) shows that there is a very small finite-size effect for early times (large $R_{g}$ ) due to the modification of the backflow by the periodic boundary conditions. However, this effect is very small and, in particular, does not affect the dependence on $\eta_{\mathrm{mb}}^{*}$. We conclude that finite-size effects are essentially negligible already for box size $L / \sigma=50$.

The late stage of the closure is determined by the competition of line tension, curvature elasticity, and membrane and solvent viscosities. It has been shown in Ref. 69 that the closure of a pore in a flat viscous, tensionless film can be described by $\eta_{\mathrm{mb}} d r_{\mathrm{po}} / d t=-\Gamma / 2$, which implies that the pore radius $r_{\mathrm{po}}$ decreases linearly in time. We have generalized this description to include the effect of curvature elasticity. In this case, the time dependence of the pore radius $r_{\mathrm{po}}$ is determined by

$$
\eta_{\mathrm{mb}}^{\mathrm{eff}} \frac{d r_{\mathrm{po}}}{d t}=-\frac{\partial F}{4 \pi \partial r_{\mathrm{po}}}=-\frac{\Gamma}{2}+\frac{\kappa}{2 R_{\mathrm{eq}}^{2}} r_{\mathrm{po}}
$$

with the radius $R_{\text {eq }}$ of the closed vesicle. The left-hand side of Eq. (22), which describes the energy dissipation, assumes a planar velocity field $v(r) \sim 1 / r$; the validity of this assumption is restricted to pore radii, which are much smaller than $R_{\text {eq }}$. The free energy $F$ of a membrane with the shape of a spherical cap is given by ${ }^{67}$

$$
F=4 \pi\left[\frac{\Gamma r_{\mathrm{po}}}{2}+\kappa\left\{1-\left(\frac{r_{\mathrm{po}}}{2 R_{\mathrm{eq}}}\right)^{2}\right\}\right] .
$$

Since a detailed theoretical description of the solvent flows through the pore and through the membrane goes beyond the scope of the present investigation, we combine all hydrodynamical effects into an effective membrane viscosity $\eta_{\mathrm{mb}}^{\text {eff }}$. The solution of Eq. (22) is

$$
r_{\mathrm{po}}(t) / R_{\mathrm{eq}}=\Gamma_{r}\left[1-\exp \left\{\left(t-t_{1}\right) / 2 \tau \Gamma_{r}\right\}\right],
$$

where $\Gamma_{r}=\Gamma R_{\mathrm{eq}} / \kappa, \tau=\eta_{\mathrm{mb}}^{\mathrm{eff}} R_{\mathrm{eq}} / \Gamma$, and $r_{\mathrm{po}}\left(t_{1}\right)=0$. The inset of Fig. 10(b) shows a comparison of the pore angle $\theta_{\mathrm{po}}$, with $\cos \left(\theta_{\mathrm{po}}\right)=1-\left(r_{\mathrm{po}} / R_{\mathrm{eq}}\right)^{2} / 2$, as obtained from simulations and Eq. (24). The functional time dependence is reproduced very well. Note, in particular, that the curvature energy slows down pore closure for larger pore radii. A fit of the simulation data yields an effective membrane viscosity $\eta_{\mathrm{mb}}^{\text {eff }}$ $=2.6\left(\eta_{\mathrm{mb}}+\eta_{0} R_{\mathrm{eq}}\right)$ (corresponding to $\left.\tau=2.6 \tau_{2}\right)$. This value is about 2.6 times larger than expected from the parameters in the simulations. We believe that this difference arises due to the effects of the hydrodynamic solvent flows via the pore 


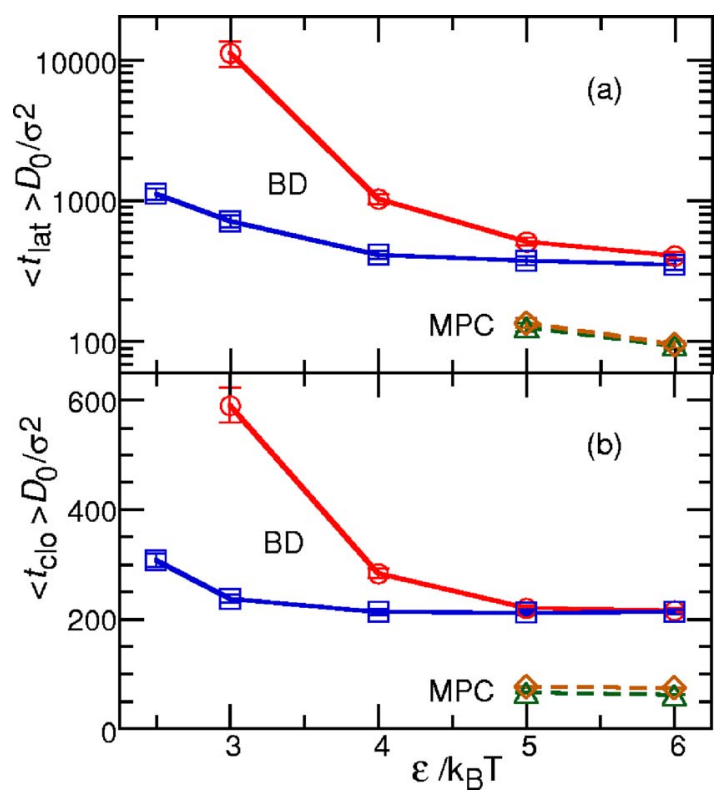

FIG. 11. (Color online) (a) Latency time $t_{\text {lat }}$ and (b) closure time $t_{\text {clo }}$ for a membrane of size $N=1000$. The full lines with symbols represent data of $\mathrm{BD}$ for $k_{\alpha} / k_{\mathrm{B}} T=5$ (squares) and $k_{\alpha} / k_{\mathrm{B}} T=10$ (circles). The dashed lines with symbols represent data of MPC for $k_{\alpha} / k_{\mathrm{B}} T=10$ with solvent viscosities $\eta_{0} \sigma^{2} / \sqrt{m_{s} k_{\mathrm{B}} T}=5.4$ (triangles) and 1.9 (diamonds).

and by membrane permeation, which have not been included in our simplified description.

Figure 11 shows both the latency time $t_{\text {lat }}$ and the closure time $t_{\text {clo }}$. We define $t_{\text {lat }}$ as the last time when the radius of gyration crosses the value $R_{g}=13.5 \sigma$ before closure; thus, the latency time is the time interval the membranes fluctuates thermally around the initial planar state before it starts its essentially deterministic path to a closed vesicle. The closure time $t_{\text {clo }}$ is the subsequent time interval until closure is completed, i.e., until $R_{g}$ reaches its equilibrium value. At small line tension $\Gamma \sim \varepsilon$, the latency time is much larger than the closure time, but the two times become comparable for large $\varepsilon$. Furthermore, we find that both times level off at large $\varepsilon$. In the case of the closure time, the reason for this behavior is the simultaneous increase of line tension and membrane viscosity, which depends approximately linearly on $\varepsilon$ (see Fig. 1 in Sec. II D). Both $t_{\text {lat }}$ and $t_{\text {clo }}$ increase with increasing bending rigidity $\kappa \sim k_{\alpha}$, since the resistance to curvature increases. This is in qualitative agreement with the prediction of Eq. (24) that the closure process is characterized by two time scales $\tau=\eta_{\mathrm{mb}}^{\text {eff }} R_{\text {eq }} / \Gamma$ and $\tau \Gamma_{r}=\eta^{\text {eff }} R_{\text {eq }}^{2} / \kappa$ related to line tension and bending rigidity, respectively.

Since the volume enclosed by the membrane deceases during vesicle formation, the solvent inside has to flow to the outside. The volume flow $J_{\text {po }}$ through a pore of radius $r_{\text {po }}$ can be estimated from a Poiseuille flow through a cylindrical capillary of this radius and length $\sigma$,

$$
J_{\mathrm{po}}=\frac{\pi r_{\mathrm{po}}^{4} \Delta P}{8 \eta_{0} \sigma},
$$

where $\Delta P$ is the pressure difference between inside and outside of the vesicle caused by the membrane dynamics. The volume flow through the membrane due to its permeability is given by

$$
J_{\mathrm{pe}}=A_{0} \Lambda \Delta P,
$$

where $A_{0}=a_{0} N$ is the area of the tensionless membrane. With decreasing pore size, the flow through the pore $J_{\text {po }}$ decreases with $r_{\text {po }}^{4}$, while the permeant flow $J_{\text {pe }}$ does not dependent on $r_{\text {po }}$. Therefore, the flow $J_{\text {po }}$ should be dominant in our simulations, with $N=1000$ and the parameters chosen in Sec. II for pore angles $\theta_{\mathrm{po}}>0.15 \pi\left(r_{\mathrm{po}}>5 \sigma\right)$. For $\theta_{\mathrm{po}}<0.15 \pi$, the permeant flow $J_{\text {pe }}$ dominates. Since the relative volume change has been already small there, the membrane permeability should not modify the closure dynamics of our simulations. Note that the threshold angle and the relative threshold pore radius, $r_{\mathrm{po}} / R_{g}$, defined by $J_{\mathrm{po}}=J_{\mathrm{pe}}$, both decrease with increasing membrane size as $N^{-1 / 4}$.

\section{VESICLE DISSOLUTION}

When the strength $\varepsilon$ of the cohesive interaction between membrane particles is sufficiently reduced, the membrane disintegrates and a vesicle dissolves. We start by simulating a vesicle with $N=1000$ in a metastable equilibrium state at $\varepsilon / k_{\mathrm{B}} T=1.5$. The interaction strength $\varepsilon / k_{\mathrm{B}} T$ is then suddenly reduced at time $t=0$. Figures 12 and 13 show an example of the dissolution dynamics. The vesicle is still metastable and keeps its initial shape for a while. Then, a pore open at $t D_{0} / \sigma^{2}=1450$ and membrane particles dissolve from the edge of the pore. Figure 13 shows that $R_{g}$ and $\alpha_{\text {sp }}$ first increase, then $R_{g}$ decreases again. The first process is opening up of a vesicle (see Fig. 12), which is the inverse process of the vesicle closure.

To separate the rupture dynamics and dissolution processes, we define the time at $R_{g}=12 \sigma$ as the rupture time $t_{\text {rup}}$. Figure 14 shows the exponential dependence of the mean rupture time $\left\langle t_{\text {rup }}\right\rangle$ on the strength $\varepsilon$ of the cohesive energy after the quench. This dependence is not modified by the hydrodynamic interactions. The line tensions are estimated from the relation $\Gamma \sigma / k_{\mathrm{B}} T=1.16 \varepsilon / k_{\mathrm{B}} T-0.22$ given in Sec. II D, which yields $\Gamma \sigma / k_{\mathrm{B}} T=1.2,1.3$, and 1.4 for $\varepsilon / k_{\mathrm{B}} T$ $=1.2,1.3$, and 1.4 , respectively.

The exponential dependence suggests that the freeenergy barrier of rupture and pore opening $\Delta F_{\mathrm{po}}$ is proportional to $\varepsilon$ and $\left\langle t_{\text {rup }}\right\rangle \propto \exp \left(\Delta F_{\text {po }} / k_{\mathrm{B}} T\right)$. Let us assume $\Delta F_{\text {po }}$ $=2 \pi r_{\mathrm{ba}} \Gamma$, where $r_{\mathrm{ba}}$ is the radius of the pore at the freeenergy barrier. We then estimate $r_{\mathrm{ba}}=3 \sigma$ from the data in Fig. 14. This is a reasonable value for the critical pore radius to apply the line-tension concept, since with a pore perimeter of about $20 \sigma$, a continuum description should be applicable. An exponential dependence of the free-energy barrier for pore opening has also been reported from micropipette-aspiration experiments. $^{24,25}$

The time development of dissolution with time origin at $R_{g}=12 \sigma$ is shown in Figs. 13 and 15. Since membranes often divide into two disjoint patches during the opening-up process, the averages in Fig. 13 are taken only for connected clusters. The sum of the particles in the first and second largest clusters is averaged in Fig. 15. The dissolution becomes faster for (i) smaller $\Gamma$ or (ii) a larger box size $L$, since the probability for dissolved particles to reassociate with membrane patches increases in smaller boxes. The dissolu- 


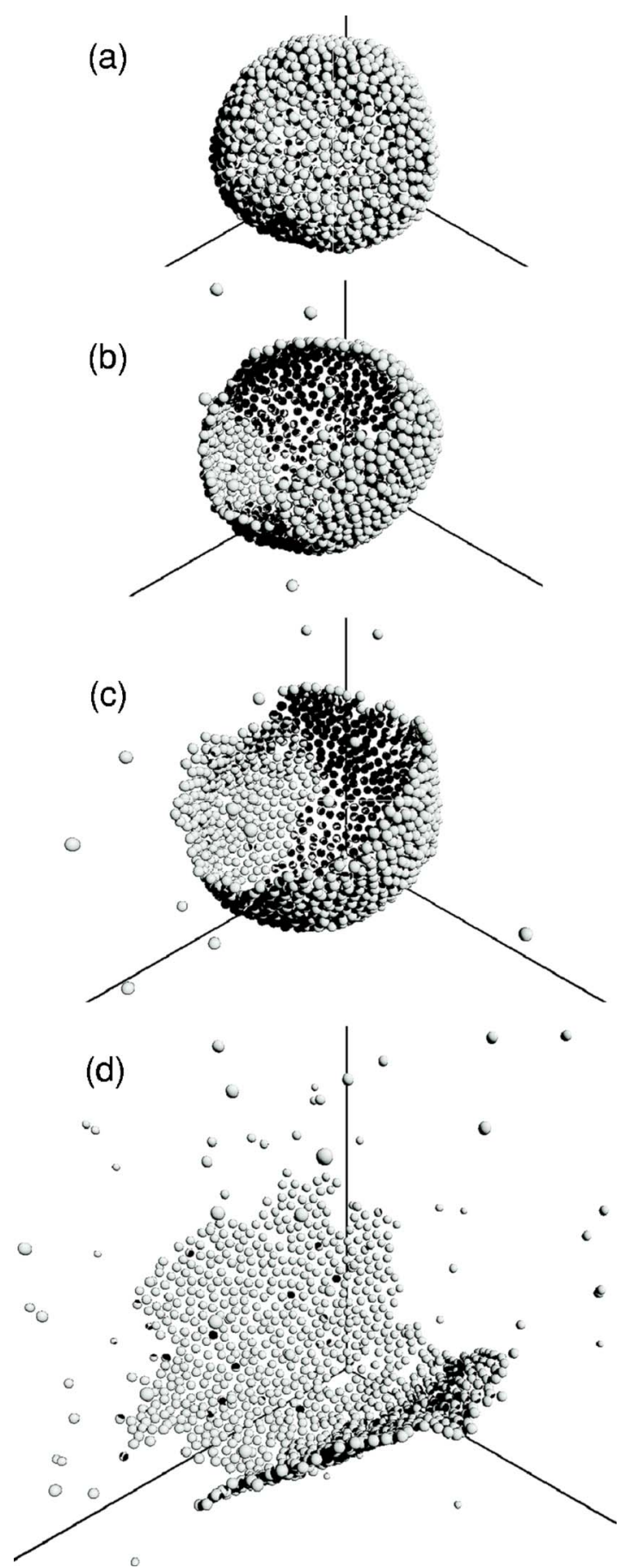

FIG. 12. Snapshots of the dissolution process of a vesicle in BD simulation with $N=1000, k_{\alpha} / k_{\mathrm{B}} T=10, \varepsilon / k_{\mathrm{B}} T=1.3$, simulation-box size $L=100 \sigma$, at times (a) $t D_{0} / \sigma^{2}=1400$, (b) $t D_{0} / \sigma^{2}=1520$, (c) $t D_{0} / \sigma^{2}=1600$, (d) $t D_{0} / \sigma^{2}$ $=2000$.

tion dynamics becomes faster with the hydrodynamic interactions but no qualitative effects are observed.

The dissolution dynamics, after the membrane has opened up to a disklike shape, can be understood from the decay equation of a circular cluster. Since particles dissolve mainly from the edge of the membrane,

$$
d N / d t=-c \sqrt{N},
$$

where the dissolution rate is proportional to $R_{\mathrm{dis}} \sim \sqrt{N}$, and not to $N$ as in the standard "radioactive" decay processes.

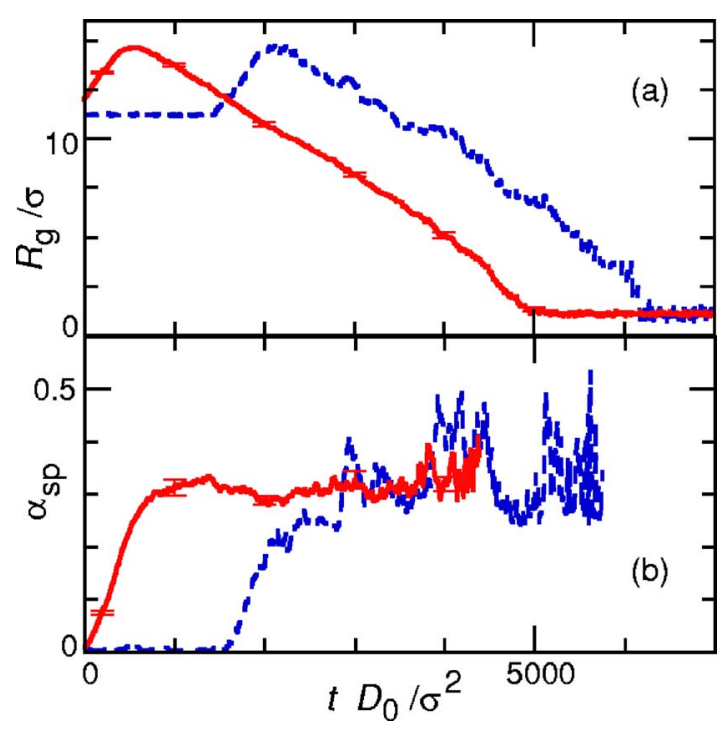

FIG. 13. (Color online) Time development of (a) the radius of gyration $R_{g}$ and (b) the asphericity $\alpha_{\mathrm{sp}}$ of the largest cluster during vesicle dissolution with $N=1000, k_{\alpha} / k_{\mathrm{B}} T=10, \varepsilon / k_{\mathrm{B}} T=1.3$, and box size $L=100 \sigma$. The dashed lines represent the same data as in Fig. 12. The solid lines represent the averaged data with the reference time set at $R_{g} / \sigma=12$. The asphericity $\alpha_{\mathrm{sp}}$ is only shown for the largest cluster, and as long as $n_{c}>50$.

Thus, the membrane size is expected to decrease as

$$
\sqrt{N(t)}=\sqrt{N_{0}}-c t / 2 \text {. }
$$

The radius $R_{g}$ of the cluster and $\sqrt{N}$ are both predicted to decrease linearly in time. These predictions agree very well with the simulation data, as shown in Figs. 13(a) and 15(a).

In fact, this agreement is surprisingly good, since a reassociation contribution has been neglected here, which reduces the dissolution in the later time development. When the reassociation is taken into account, the dissolution dynamics is described by

$$
d N / d t=-c \sqrt{N}+b \sqrt{N}\left(N_{0}-N\right) .
$$

The reassociation rate is proportional to the edge length and thus $\sqrt{N}$, and to the monomer number in solution, $N_{0}-N$. Thus, dissolution becomes slower in the late stage,

$$
N(t)=N_{\mathrm{eq}} / \tanh ^{2}\left(b \sqrt{N_{\mathrm{eq}}} t / 2+d\right),
$$

where $N_{\mathrm{eq}}=N_{0}-c / b$ and $d=a \tanh \left(\sqrt{N_{\mathrm{eq}} / N_{0}}\right)$. In the simulations, the effect of reassociation is clearly seen only in the

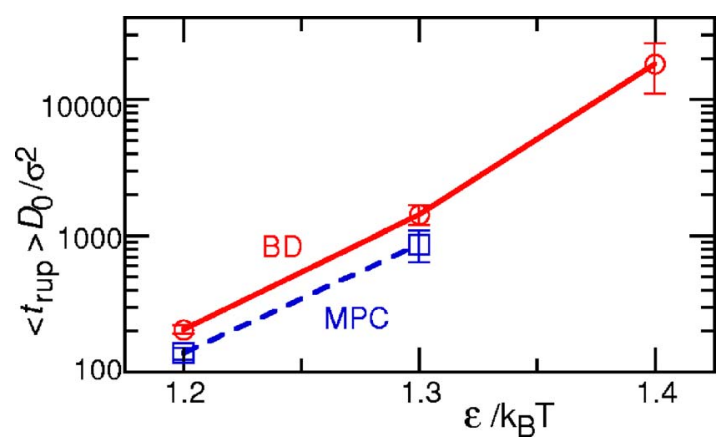

FIG. 14. (Color online) Rupture time $t_{\text {rup }}$ of a vesicle with $N=1000$ and $k_{\alpha} / k_{\mathrm{B}} T=10$. The circles and squares represent data of $\mathrm{BD}$ and MPC, respectively. 

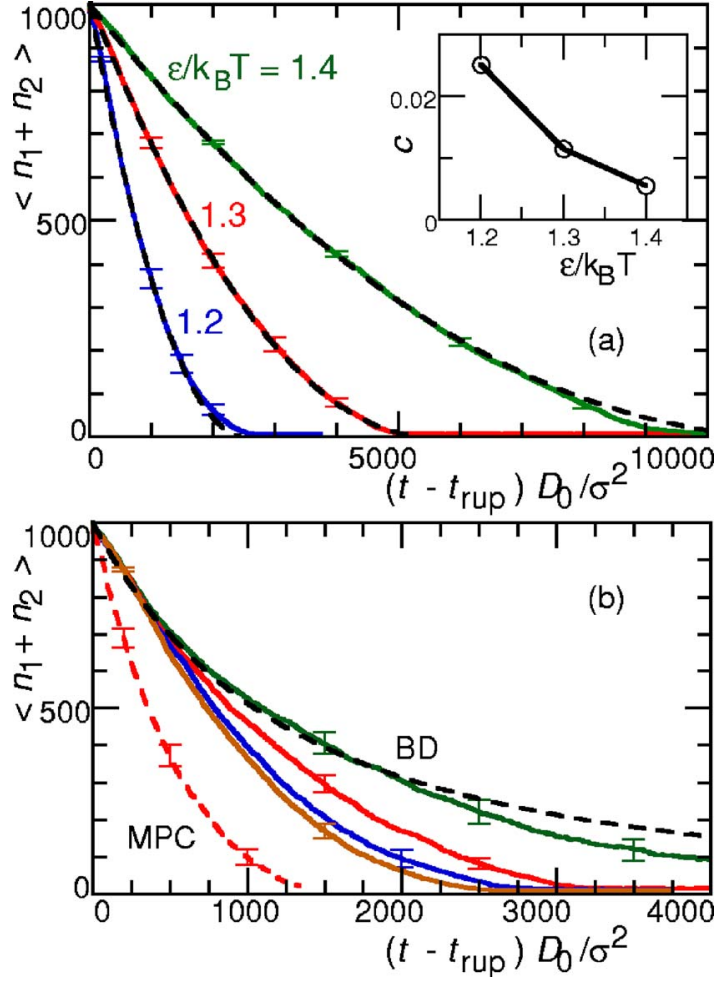

FIG. 15. (Color online) Time development of the average number $\left\langle n_{1+2}\right\rangle$ of particles in the first and second largest clusters, for $N=1000$ and $k_{\alpha} / k_{\mathrm{B}} T$ $=10$. (a) BD results for $\varepsilon / k_{\mathrm{B}} T=1.2,1.3$, and 1.4 , with box size $L / \sigma=100$. The dashed lines are fits to Eq. (28). The inset shows the $\varepsilon$ dependence of the decay rate $c$ in Eq. (28). (b) The solid lines represent results of BD simulations with $L / \sigma=40,50,64$, and 100 (from top to bottom) at $\varepsilon / k_{\mathrm{B}} T$ $=1.2$. The short-dashed (red) line represents results of MPC simulations for $L / \sigma=50$ and $\varepsilon / k_{\mathrm{B}} T=1.2$. The long-dashed line (black) is obtained from Eq. (30) with $N_{\text {eq }}=10, N_{0}=1000$, and the same decay rate $c$ as determined in (a). Error bars are shown at several data points.

smallest cell with $L / \sigma=40$, as shown in Fig. 15(b). On the other hand, membranes often divide into disjoint patches. This enhances the dissolution and reduces the effects of the reassociation.

A similar opening-up dynamics was observed experimentally in the lysis of liposomes after the addition of detergents. ${ }^{21}$ The detergents likely remove lipids mainly from the edges of a membrane. This process can be understood as a reduction of line tension $\Gamma$ by the detergents. Other dissolution-dynamics processes were also reported in Ref. 21, depending on the combination of the lipids and detergents. More detailed studies with molecular models are necessary to obtain a better understanding of these processes during lysis.

\section{SUMMARY}

We have studied the self-assembly and dissolution dynamics of membranes, both with and without hydrodynamic interactions. In the self-assembly process, membrane particles aggregate to form discoidal clusters, which then coalesce into large clusters. The clusters growth in this regime is found to follow a linear time dependence, both with and without hydrodynamic interactions. We have shown that this behavior can be understood on the basis of Smoluchowski rate equations. When the clusters are sufficiently large, they form vesicles via a bowl-like shape.

Vesicle dissolution after a quench to lower line tensions starts by the opening of a pore in the membrane. The rupture time is found to increase exponentially with increasing line tension. The membrane then flattens out and particles dissociate at its edge. This is found to lead to a linear decrease of the radius of the membrane patch in time.

The hydrodynamic interactions affect the time scales of cluster aggregation and vesicle dissolution. In both cases, the processes are sped up by hydrodynamics. A particularly interesting behavior is observed for vesicle closure. Two regimes are found, an initial regime where the dynamics is governed by the solvent viscosity, and a later regime where the membrane viscosity becomes important. Furthermore, hydrodynamics also affects the membrane shapes during closure, by making the vesicles more spherical.

We have demonstrated here that the combination of a meshless-membrane model with multiparticle collision dynamics for the solvent is very useful, since the hydrodynamic interactions can easily switch on or off in the simulations. Static equilibrium properties can be investigated very efficiently by Brownian dynamics or Monte Carlo simulations, since they need much less computational time than simulations involving hydrodynamic interactions. Thus, our membrane model is very well suited to study other dynamical membrane phenomena, such as phase separation and budding of multicomponent vesicles or vesicle deformation under flow.

\section{ACKNOWLEDGMENT}

Stimulating discussions with S. Egelhaaf (Düsseldorf) are gratefully acknowledged.

${ }^{1}$ S. A. Safran, Statistical Thermodynamics of Surfaces, Interfaces, and Membranes (Addison-Wesley, Reading, MA, 1994).

${ }^{2}$ G. Gompper and M. Schick, in Self Assembling Amphiphilic Systems, Phase Transitions and Critical Phenomena Vol. 16 (Academic, London, 1994).

${ }^{3}$ Structure and Dynamics of Membranes, edited by R. Lipowsky and E. Sackmann (Elsevier Science, Amsterdam, 1995).

${ }^{4}$ U. Seifert, Adv. Phys. 46, 13 (1997).

${ }^{5}$ H. Hotani, F. Nomura, and Y. Suzuki, Curr. Opin. Colloid Interface Sci. 4, 358 (1999).

${ }^{6}$ M. Kraus, W. Wintz, U. Seifert, and R. Lipowsky, Phys. Rev. Lett. 77, 3685 (1996).

${ }^{7}$ K. H. de Haas, C. Blom, D. van den Ende, M. H. G. Duits, and J. Mellema, Phys. Rev. E 56, 7132 (1997).

${ }^{8}$ H. Noguchi and G. Gompper, Phys. Rev. Lett. 93, 258102 (2004).

${ }^{9}$ J. Beaucourt, F. Rioual, T. Séon, T. Biben, and C. Misbah, Phys. Rev. E 69, 011906 (2004).

${ }^{10}$ O. Diat and D. Roux, J. Phys. II 3, 9 (1993).

${ }^{11}$ K. Mortensen, Curr. Opin. Colloid Interface Sci. 6, 140 (2001)

${ }^{12}$ F. Nettersheim, J. Zipfel, U. Olsson, F. Renth, P. Lindner, and W. Richtering, Langmuir 19, 3603 (2003).

${ }^{13}$ M. Gradzielski, Curr. Opin. Colloid Interface Sci. 8, 337 (2003).

${ }^{14}$ J. Leng, S. U. Egelhaaf, and M. E. Cates, Europhys. Lett. 59, 311 (2002).

${ }^{15}$ J. Leng, S. U. Egelhaaf, and M. E. Cates, Biophys. J. 85, 1624 (2003).

${ }^{16}$ T. M. Weiss, T. Narayanan, C. Wolf, M. Gradzielski, P. Panine, S. Finet, and W. I. Helsby, Phys. Rev. Lett. 94, 038303 (2005).

${ }^{17}$ K. Bryskhe, S. Bulut, and U. Olsson, J. Phys. Chem. B 109, 9265 (2005).

${ }^{18}$ A. M. Seddon, P. Curnow, and P. J. Booth, Biochim. Biophys. Acta 1666, 105 (2004).

${ }^{19}$ O. López, A. de la Maza, L. Coderch, C. López-Iglesias, E. Wehrli, and J. L. Parra, FEBS Lett. 426, 314 (1998). 
${ }^{20}$ U. Kragh-Hansen, M. le Maire, and J. V. Moller, Biophys. J. 75, 2932 (1998).

${ }^{21}$ F. Nomura, M. Nagata, T. Inaba, H. Hiramatsu, H. Hotani, and K. Takiguchi, Proc. Natl. Acad. Sci. U.S.A. 98, 2340 (2001).

${ }^{22}$ O. Sandre, L. Moreaux, and F. Brochard-Wyart, Proc. Natl. Acad. Sci. U.S.A. 96, 10591 (1999).

${ }^{23}$ E. Karatekin, O. Sandre, H. Guitouni, N. Borghi, P. H. Puech, and F. Brochard-Wyart, Biophys. J. 84, 1734 (2003).

${ }^{24}$ E. A. Evans and F. Ludwig, J. Phys.: Condens. Matter 12, A315 (2000).

${ }^{25}$ E. A. Evans, V. Heinrich, F. Ludwig, and W. Rawicz, Biophys. J. 85, 2342 (2003)

${ }^{26}$ H. V. Ly and M. L. Longo, Biophys. J. 87, 1013 (2004).

${ }^{27}$ G. Gompper and D. M. Kroll, in Statistical Mechanics of Membranes and Surfaces, 2nd ed., edited by D. R. Nelson, T. Piran, and S. Weinberg (World Scientific, Singapore, 2004).

${ }^{28}$ G. Gompper and D. M. Kroll, J. Phys.: Condens. Matter 9, 8795 (1997).

${ }^{29}$ D. H. Boal and M. Rao, Phys. Rev. A 46, 3037 (1992).

${ }^{30}$ S. J. Zhao and J. T. Kindt, Europhys. Lett. 69, 839 (2005).

${ }^{31}$ S. E. Feller, Curr. Opin. Colloid Interface Sci. 5, 217 (2000).

${ }^{32}$ S. J. Marrink, E. Lindahl, O. Edholm, and A. E. Mark, J. Am. Chem. Soc. 123, 8638 (2001).

${ }^{33}$ S. O. Nielsen, C. F. Lopez, G. Srinivas, and M. L. Klein, J. Phys.: Condens. Matter 16, R481 (2004).

${ }^{34}$ B. Smit, P. A. J. Hilbers, K. Esselink, L. A. M. Rupert, N. M. van Os, and A. G. Schlijper, Nature (London) 348, 624 (1990).

${ }^{35}$ R. Goetz, G. Gompper, and R. Lipowsky, Phys. Rev. Lett. 82, 221 (1999).

${ }^{36}$ S. A. Shkulipa, W. K. den Otter, and W. J. Briels, Biophys. J. 89, 823 (2005).

${ }^{37}$ S. Yamamoto, Y. Maruyama, and S. A. Hyodo, J. Chem. Phys. 116, 5842 (2002).

${ }^{38}$ H. Noguchi and M. Takasu, Phys. Rev. E 64, 041913 (2001).

${ }^{39}$ I. R. Cooke and M. Deserno, J. Chem. Phys. 123, 224710 (2005).

${ }^{40}$ O. Farago, N. Gronbech-Jensen, and P. Pincus, Phys. Rev. Lett. 96, 018102 (2006).

${ }^{41}$ G. Brannigan, L. C. L. Lin, and F. L. H. Brown, Eur. Biophys. J. 35, 104 (2006).

${ }^{42}$ H. Noguchi and G. Gompper, Phys. Rev. E 73, 021903 (2006).
${ }^{43}$ J. M. Drouffe, A. C. Maggs, and S. Leibler, Science 254, 1353 (1991).

${ }^{44}$ T. Belytschko, Y. Krongauz, D. Organ, M. Fleming, and P. Krysl, Comput. Methods Appl. Mech. Eng. 139, 3 (1996).

${ }^{45}$ P. Lancaster and K. Salkaskas, Math. Comput. 37, 141 (1981).

${ }^{46}$ A. Malevanets and R. Kapral, J. Chem. Phys. 110, 8605 (1999).

${ }^{47}$ A. Malevanets and J. M. Yeomans, Europhys. Lett. 52, 231 (2000).

${ }^{48}$ M. Ripoll, K. Mussawisade, R. G. Winkler, and G. Gompper, Europhys. Lett. 68, 106 (2004).

${ }^{49}$ H. Noguchi and G. Gompper, Proc. Natl. Acad. Sci. U.S.A. 102, 14159 (2005).

${ }^{50}$ S. H. Lee and R. Kapral, J. Chem. Phys. 124, 214901 (2006).

${ }^{51}$ T. Ihle and D. M. Kroll, Phys. Rev. E 63, 020201(R) (2001).

${ }^{52}$ N. Kikuchi, C. M. Pooley, J. F. Ryder, and J. M. Yeomans, J. Chem. Phys. 119, 6388 (2003).

${ }^{53}$ J. T. Padding and A. A. Louis, Phys. Rev. Lett. 93, 220601 (2004).

${ }^{54}$ M. P. Allen and D. J. Tildesley, Computer Simulation of Liquids (Clarendon, Oxford, 1987)

${ }^{55}$ W. Helfrich, Z. Naturforsch. C 28, 693 (1973).

${ }^{56}$ F. Jülicher and R. Lipowsky, Phys. Rev. E 53, 2670 (1996).

${ }^{57}$ T. Umeda and U. Suezaki, Phys. Rev. E 71, 011913 (2005)

${ }^{58}$ In Sec. II C of Ref. 42, the factor 2 should be removed.

${ }^{59}$ W. Cai and T. C. Lubensky, Phys. Rev. E 52, 4251 (1995).

${ }^{60}$ H. Furukawa, Adv. Phys. 34, 703 (1985).

${ }^{61}$ A. J. Bray, Adv. Phys. 43, 357 (1994).

${ }^{62}$ P. I. Hurtado, J. Marro, and E. V. Albano, Europhys. Lett. 59, 14 (2002).

${ }^{63}$ N. Yoshioka, I. Varga, F. Kum, S. Yukawa, and N. Ito, Phys. Rev. E 72, 061403 (2005).

${ }^{64}$ W. B. Russel, D. A. Saville, and W. E. Schowalter, Colloidal Dispersions (Cambridge University Press, Cambridge, 1991).

${ }^{65}$ D. F. Evans and H. Wennerström, The Colloidal Domain: Where Physics, Chemistry, Biology, and Technology Meet (Wiley-VCH, New York, 1994).

${ }^{66}$ R. C. Ball, D. A. Weitz, T. A. Witten, and F. Leyvraz, Phys. Rev. Lett. 58, 274 (1987).

${ }^{67}$ P. Fromherz, Chem. Phys. Lett. 94, 259 (1983).

${ }^{68}$ J. Rudnick and G. Gaspari, J. Phys. A 19, L191 (1986).

${ }^{69}$ G. Debrégeas, P. Martin, and F. Brochard-Wyart, Phys. Rev. Lett. 75, 3886 (1995) 